\title{
Pseudospectral Collocation Methods for the Computation of the Self-Force on a Charged Particle: Generic Orbits around a Schwarzschild Black Hole
}

\author{
Priscilla Canizares, ${ }^{1}$ Carlos F. Sopuerta, ${ }^{1}$ and José Luis Jaramillo ${ }^{2,3}$ \\ ${ }^{1}$ Institut de Ciències de l'Espai (CSIC-IEEC), Facultat de Ciències, \\ Campus UAB, Torre C5 parells, E-08193 Bellaterra, Spain \\ ${ }^{2}$ Max Planck Institute für Gravitationsphysik, Albert-Einstein-Institute, 14476 Postdam, Germany \\ ${ }^{3}$ Laboratoire Univers et Théories (LUTH), Observatoire de Paris, CNRS, \\ Université Paris Diderot, 5 place Jules Janssen, 92190 Meudon, France
}

(Dated: October 25, 2018)

\begin{abstract}
The inspiral of a stellar compact object into a massive black hole, an extreme-mass-ratio inspiral, is one of the main sources of gravitational waves for the future space-based Laser Interferometer Space Antenna. We expect to be able to detect and analyze many cycles of these slowly inspiraling systems, which makes them truly high precision tools for gravitational-wave astronomy. To that end, the use of very precise theoretical waveform templates in the data analysis is required. To build them we need to have a deep understanding of the gravitational backreaction mechanism responsible for the inspiral. The self-force approach describes the inspiral as the action of a local force that can be obtained from the regularization of the perturbations created by the stellar compact object on the massive black hole geometry. In this paper we extend a new time-domain technique for the computation of the self-force from the circular case to the case of eccentric orbits around a non-rotating black hole. The main idea behind our scheme is to use a multidomain framework in which the small compact object, described as a particle, is located at the interface between two subdomains. Then, the equations at each subdomain are homogeneous wave-type equations, without distributional sources. In this particle-without-particle formulation, the solution of the equations is smooth enough to provide good convergence properties for the numerical computations. This formulation is implemented by using a pseudospectral collocation method for the spatial discretization, combined with a Runge Kutta algorithm for the time evolution. We present results from several simulations of eccentric orbits in the case of a scalar charged particle around a Schwarzschild black hole, an excellent testbed model for testing the techniques for self-force computations. In particular, we show the convergence of the method and its ability to resolve the field and its derivatives across the particle location. Finally, we provide numerical values of the self-force for different orbital parameters.
\end{abstract}

PACS numbers: 04.30.Db, 04.40.Dg, 95.30.Sf, 97.10.Sj

\section{INTRODUCTION}

One of the main subjects of activity in the emergent area of Gravitational Wave Astronomy is the physical description of the different astrophysical and cosmological sources of gravitational radiation that are expected to be detected by the present generation (LIGO [1, GEO600 [2, VIRGO [3]) and future/planned (LCGT [4], LISA [5], ET [6], etc.) laser interferometer observatories. The description of sources of gravitational waves plays an important role for several reasons. On the one hand, we need to understand well these sources in order to convert the information from their observations in gravitational waves (and perhaps in some electromagnetic bands) into new scientific knowledge. On the other hand, in order to detect and obtain accurate physical information from these observations we need to use data analysis techniques, like the matched filter, that require the use of precise waveform templates in order to separate the signals from the noise and to estimate appropriately the source parameters.

In the case of the future Laser Interferometer Space Antenna (LISA) [5] (see [7, 8] for the proceedings of the 7th international LISA symposium), the main expected sources of gravitational waves are: Galactic com- pact binaries, mergers of Massive Black Holes (MBHs), the inspirals of stellar-mass compact objects into MBHs, and stochastic gravitational-wave backgrounds of diverse origin. This paper is devoted to the description of the dynamics of the third type of source which, due to the extreme mass ratios involved, are usually known as Extreme-Mass-Ratio Inspirals (EMRIs). The EMRIs of interest for LISA are associated with relaxed galactic nuclei that have a dense stellar population (a stellar cusp or core) around a hypothetical central MBH. In this situation, there is a non-negligible probability for a stellarmass compact object (SCO), with masses in the range $m \sim 1-50 M_{\odot}$, to end up in a slow inspiral towards the MBH (see also [9] for details and references on the astrophysics of EMRIs). The range of the MBH mass of interest for LISA is $M \sim 10^{4}-10^{7} M_{\odot}$, which means that the mass ratios involved in EMRIs are in the interval $\mu=m / M \sim 10^{-7}-10^{-3}$.

In brief, the situation we face in trying to detect EMRIs with LISA is the following: They are systems that during the last year of inspiral before plunge complete of the order of $10^{5}$ cycles [10, so they fall into the $\sim 0.1 \mathrm{mHz}$ part of the LISA band. Therefore, the mass ratio implies not only the existence of very different spatial scales (e.g. the size of the SCO as compared to the size of the 
$\mathrm{MBH})$, but also of two very different time scales, namely the orbital time scale versus the inspiral time scale, so that $T_{\text {orbital }} / T_{\text {inspiral }} \sim \mu=m / M$. Despite the difficulties introduced by the extreme mass ratios involved in the problem, there is an advantage to it: we can assume that the spacetime geometry is the one due to the $\mathrm{MBH}$ with deviations produced by the SCO. Then, one can use perturbation theory to deal with EMRIs. In this context, the inspiral can be described as the action of a local force, the so-called self-force, according to the equation of motion derived in [11, 12, known as the MiSaTaQuWa equation of motion. The MiSaTaQuWa equation can be used as the foundations of a self-consistent scheme to simulate an EMRI event in an adiabatic way by coupling it to the Partial Differential Equations (PDEs) describing the perturbations produced by the SCO (together with a scheme to identify the regular part of the perturbations responsible for the inspiral). For recent studies on these issues see [13 15. For reviews on different aspects of EMRIs see [16 18].

Given the amount of cycles required for EMRIs and their computational cost we cannot expect to generate complete waveform template banks by means of full selfforce calculations. Instead, the goal of these studies should be to understand all the details of the structure of the self-force so that we can formulate efficient and precise algorithms to create the waveforms needed for LISA data analysis, perhaps complementing present approximate schemes [19-22].

Moreover, due to the large number of cycles that will be available in future LISA EMRI observations, we expect to determine the EMRI physical parameters with high precision [19]. Given also that the expected event rate is in the range $10-10^{3} \mathrm{EMRI} / \mathrm{yr}$ 23, 24] up to a redshift $z \approx 1$, these observation will have an important impact not only on astrophysics (see [9] and references therein), but also on cosmology and fundamental physics [25], from precise measurements of cosmological parameters [26, 27] to tests of the geometry of MBHs 28 34 and/or the gravitational interaction [35, 36].

If Intermediate-Mass Black Holes (IMBHs) [37, 38] exist (with masses in the range $10^{2}-10^{4} M_{\odot}$ ), as several recent observations suggest, they can form binaries with intermediate mass ratios resulting in the so-called Intermediate-Mass-Ratio Inspirals (IMRIs). LISA will be sensitive to one type of IMRIs: That an IMBH into a $\mathrm{MBH}$. In contrast, the inspiral of an SCO into an IMBH, another type of IMRIs outside the LISA band, is of interest for the next generations of ground-based detectors 39, 40. We can obtain an approximate description (probably not accurate enough for data analysis purposes) of IMRIs by using the same methods that are used for EMRIs.

In the calculation of the self-force, it is reasonable to neglect the structure of the $\mathrm{SCO}$ and describe it as a point-like object. This means that the retarded metric pertubations generated by the SCO diverge at the particle location, and hence they need to be regularized. An algorithm that has been widely employed is the mode sum regularization scheme [41 48]. It substracts, mode by mode (harmonic $\ell$-modes) from the retarded field, the singular part of the perturbations that does not contribute to the self-force. We therefore need a method for computing the different multipoles of the retarded part of the metric perturbations induced by the SCO. Due to the complexity of these linear equations, solutions can only be obtained numerically, either using frequency- or time-domain methods. Both have advantages and disadvantages. Because EMRIs are expected to have high eccentricities and frequency-domain methods have shown difficulties in this case, we concentrate on time-domain methods. Time-domain methods have difficulties related to the resolution of the SCO (see, e.g. 49]), since many of them need to introduce an artificial scale in order to model the distributional energy-momentum tensor that describes the SCO.

To solve the problems related to the artificial scale associated with the SCO some new techniques have been recently proposed [50 53] which are similar to the puncture method introduced in numerical relativity [54 56]. However, they do not completely solve the problem of the spatial resolution associated with the source terms. We recently introduced [57, 58] a new technique, for the case of circular orbits, that completely avoids the introduction of a spatial scale. The method, described in detail in 58 (henceforth Paper I), is based on the splitting of the spatial computational domain (the one-dimensional domain associated with the radial coordinate) into several subdomains, in such a way that the SCO is located at the interface of two such subdomains. For the circular case, the SCO is at rest in the spatial domain. The outcome of this setup is that the equations at each subdomain are homogeneous wave-like equations. In this way, we ensure the smoothness of the solutions in each domain and at the same time we avoid artificial scales associated with the SCO. The SCO appears in the boundary/matching conditions between the subdomains that surround it. This setup is ideal to use high-precision numerical methods, which normally require a high degree of smoothness in the solution. In Paper I we implemented these ideas for the case of a charged scalar particle in circular orbits around a non-rotating MBH using the PseudoSpectral Collocation (PSC) method for the spatial discretization (a similar technique has also been proposed recently in [59]). In this sense, the smoothness of the solutions of the homogeneous wave-like equations involved, ensures the exponential convergence of the method, as shown in Paper I. This study has further demonstrated the high precision of the method by comparing results with previous calculations of the self-force on a charged particle in circular motion [48, 60, 61. Finally, it was shown that by adjusting the subdomains and the number of collocation points, one also obtains an efficient method (in terms of computational time) for the calculation of the self-force. These techniques can be directly imported to the gravitational (and electromagnetic) case. 
The main aim of the present paper is to show that the techniques shown in Paper I can be extended to the case of generic eccentric orbits. The key ingredient of our formulation, which we call the Particle-without-Particle formulation, is to divide the computational domain into subdomains and to locate the particle at the interface of two of them in such a way that the equations do not have a distributional term that deteriorates the smoothness of the solution. The challenge is to ensure communication between the domains in an appropriate way to take into account the particle effects in a precise way. The main challenge in comparison with Paper I is to adapt these techniques to the case where the particle is no longer at rest in the spatial domain, as its radial coordinate is changing with time. This will be achieved by adapting the mapping between the physical domain (corresponding to the radial coordinate) and the spectral domain (corresponding to the coordinate that labels the collocation points used in the PSC method) such that the particle is moving in the physical domain but it is at rest in the spectral domain. In this way, we can apply all the machinery shown in Paper I. Here we present the ingredients of the new formulation in order to handle eccentric orbits: the equations, the junction conditions, different ways of implementing it numerically, etc. In particular we show that the spectral convergence is preserved. We also show that we can evolve the system in time with long-term stability and a good resolution of the jumps in the derivatives of the solution induced by the presence of the particle. In particular, we provide values of the selfforce components, at the pericenter radius, for different eccentric orbits.

The organization of this paper is as follows: In Sec. III we introduce the formulation of the physical model, a charged scalar particle orbiting a Schwarzschild MBH, including the basic formulae for the computation of the self-force via the mode-sum regularization scheme. In Sec. III] we introduce the basic mathematical formulation of our method, based on the division of the physical domain into subdomains and on the communication between them. In Sec. IV we describe how to implement the mathematical formulation of Sec. III using the PSC method. In Sec. V we show the performance of the numerical codes that we have designed to implement the new scheme as well as results from the computation of the self-force. We conclude with a summary and discussion in Sec.VI, including future work. Three appendices complete this paper: In Appendix A we summarize the main equations that describe the geodesic motion that we use in this work. In Appendix B we provide the main formulae related to the structure of the singular field and the associated regularization parameters. Finally, in Appendix C] we just provide the expressions for the time derivatives of the jumps in the characteristic variables. Throughout this paper we use the metric signature $(-,+,+,+)$ and geometric units in which $G=c=1$.

\section{CHARGED SCALAR PARTICLE ORBITING A SCHWARZSCHILD MBH}

In order to simplify the presentation of our techniques we use a simplified EMRI model, corresponding to a charged scalar particle orbiting a non-rotating black hole. In this model the SCO is represented as a particle, with charge $q$ associated to a scalar field $\Phi$, and the $\mathrm{MBH}$ is described by a fixed spacetime geometry, in the sense that it is not affected by the charged particle. Since we are interested in the dynamics of EMRIs in General Relativity, the geometry of the non-rotating $\mathrm{MBH}$ is given by the Schwarzschild spacetime:

$$
d s^{2}=f\left(-d t^{2}+d r^{* 2}\right)+r^{2} d \Omega^{2}, \quad f=1-\frac{2 M}{r},
$$

where $M$ is the MBH mass, $d \Omega^{2}=d \theta^{2}+\sin ^{2} \theta d \varphi^{2}$ denotes the metric of the two-sphere, and

$$
r^{*}=r+2 M \ln \left(\frac{r}{2 M}-1\right),
$$

is the so-called radial tortoise coordinate. The particle induces the scalar field $\Phi$ satisfying the following wavelike equation (see, e.g. [17]):

$$
\mathrm{g}^{\alpha \beta} \nabla_{\alpha} \nabla_{\beta} \Phi(x)=-4 \pi q \int_{\gamma} d \tau \delta_{4}(x, z(\tau)),
$$

where $\mathrm{g}^{\alpha \beta}$ denotes the Schwarzschild inverse metric (1) and $\nabla_{\mu}$ the associated canonical connection; $\tau$ denotes proper time associated with the particle along its timelike worldline $\gamma$, with coordinates $x^{\mu}=z^{\mu}(\tau)$ and $\delta_{4}\left(x, x^{\prime}\right)$ is the invariant Dirac biscalar distributional functional in Schwarzschild spacetime, defined as

$$
\left(\delta_{4}, f\right)(x) \equiv \int d^{4} x^{\prime} \sqrt{-\mathrm{g}\left(x^{\prime}\right)} f\left(x^{\prime}\right) \delta_{4}\left(x^{\prime}, x\right)=f(x),
$$

with $\mathrm{g}$ being the metric determinant.

The field equation (3) has to be complemented with the equation of motion for the scalar charged particle:

$$
m \frac{d u^{\mu}}{d \tau}=F^{\mu}=\left.q\left(\mathrm{~g}^{\mu \nu}+u^{\mu} u^{\nu}\right)\left(\nabla_{\nu} \Phi\right)\right|_{\gamma}, u^{\mu}=\frac{d z^{\mu}}{d \tau},
$$

where $m$ and $u^{\mu}$ are the mass and 4-velocity of the particle, respectively. The coupled set of equations formed by the Partial Differential Equation (PDE) for the scalar field (3) and the Ordinary Differential Equation (ODE) for the particle trajectory (5) constitute our testbed model for an EMRI. The SCO generates scalar field according to (3), which in turn, affects to the SCO motion according to (5), that is, through the action of a local force. This mechanism is the analogous of the gravitational backreaction mechanism that produces the inspiral via the gravitational self-force.

However, this is not the end of the story since the retarded solution of (3) is singular at the particle location, whereas the force in Eq. (5) involves the gradient of the 
field evaluated at the particle location. Therefore, as they stand, Eqs. (3) and (5) are formal equations that require an appropriate regularization to become fully meaningful. Following 62, the retarded field can be split into two parts: a singular piece, $\Phi^{\mathrm{S}}$, which contains the singular structure of the field and satisfies the same wave equation as the retarded field, i.e. Eq. (3), and a regular part, $\Phi^{\mathrm{R}}$, that satisfies the homogeneous equation associated with Eq. (3). As it turns out, $\Phi^{\mathrm{R}}$ is regular and differentiable at the particle's position and is the solely responsible of the scalar self-force 62. We can therefore write

$$
F_{\mathrm{R}}^{\mu}=\left.q\left(\mathrm{~g}^{\mu \nu}+u^{\mu} u^{\nu}\right)\left(\nabla_{\nu} \Phi^{\mathrm{R}}\right)\right|_{\gamma},
$$

which gives a definite sense to the equations of motion of the system.

In solving the field equation (3) we can make use of the spherical symmetry of the $\mathrm{MBH}$ geometry to expand the solution in scalar spherical harmonics (see Paper I for conventions):

$$
\Phi=\sum_{\ell=0}^{\infty} \sum_{m=-\ell}^{\ell} \Phi^{\ell m}(t, r) Y^{\ell m}(\theta, \varphi)
$$

The advantage of this expansion is that each harmonic mode, $\Phi^{\ell m}(t, r)$, is decoupled from the rest and satisfies the following $1+1$ wave-type equation:

$$
\left\{-\frac{\partial^{2}}{\partial t^{2}}+\frac{\partial^{2}}{\partial r^{* 2}}-V_{\ell}(r)\right\}\left(r \Phi^{\ell m}\right)=S^{\ell m} \delta\left(r-r_{p}(t)\right)
$$

where

$$
V_{\ell}(r)=f(r)\left[\frac{\ell(\ell+1)}{r^{2}}+\frac{2 M}{r^{3}}\right],
$$

is the Regge-Wheeler potential for scalar fields on the Schwarzschild geometry and

$$
S^{\ell m}=-\frac{4 \pi q f\left(r_{p}\right)}{r_{p} u^{t}} \bar{Y}^{\ell m}\left(\frac{\pi}{2}, \varphi_{p}\right),
$$

is the coefficient of the singular source term [76] due to the presence of the particle, and the bar denotes complex conjugation. Here we have assumed, without loss of generality, that the particle's orbit takes place in the equatorial plane $\theta=\pi / 2$. Moreover, $r_{p}$ and $\varphi_{p}$ denote the radial and azimuthal coordinates of the particle, and are functions of the coordinate time $t$.

The expansion in spherical harmonics is useful not only for simplifying the construction of the retarded solution, but also in terms of constructing its regular part, $\Phi^{\mathrm{R}}$. Indeed, it turns out that each harmonic mode of the retarded field, $\Phi^{\ell m}(t, r)$, is finite and continuous at the location of the particle. It is the sum over $\ell$ what diverges there. Here is where the mode-sum regularization scheme [41, 45, 46] comes into play, as it provides analytic expressions for the singular part of each $\ell$-mode of the retarded field. These expressions for the singular field are valid only near the particle location. The regularized self-force is thus obtained by computing numerically each harmonic mode of the self-force and subtracting the singular part provided by the mode-sum scheme. Thus, the regular part of the gradient of the field, $\nabla_{\alpha} \Phi^{\mathrm{R}} \equiv \Phi_{\alpha}^{\mathrm{R}}$, is given by

$$
\Phi_{\alpha}^{\mathrm{R}}\left(z^{\mu}(\tau)\right)=\lim _{x^{\mu} \rightarrow z^{\mu}(\tau)} \sum_{\ell=0}^{\infty}\left(\Phi_{\alpha}^{\ell}\left(x^{\mu}\right)-\Phi_{\alpha}^{\mathrm{S}, \ell}\left(x^{\mu}\right)\right) .
$$

Introducing,

$$
\Phi_{\alpha}^{\ell}\left(x^{\mu}\right)=\sum_{m=-\ell}^{\ell} \nabla_{\alpha}\left(\Phi^{\ell m}(t, r) Y^{\ell m}(\theta, \varphi)\right),
$$

the structure of the singular field can can be written as:

$$
\begin{aligned}
\Phi_{\alpha}^{\mathrm{S}, \ell} & =q\left[\left(\ell+\frac{1}{2}\right) A_{\alpha}+B_{\alpha}+\frac{C_{\alpha}}{\ell+\frac{1}{2}}\right. \\
& \left.+\frac{D_{\alpha}}{\left(\ell-\frac{1}{2}\right)\left(\ell+\frac{3}{2}\right)}+\ldots\right] .
\end{aligned}
$$

The expressions for the regularization parameters, $A_{\alpha}$, $B_{\alpha}, C_{\alpha}$, and $D_{\alpha}$, can be found in the literature for generic orbits [46, 48, 63, 64. They do not depend on $\ell$ but depend on the trajectory of the particle. The three first coefficients of (13) are responsible for the divergences, whereas the remaining terms converge to zero once they are summed over $\ell$. The expressions for the regularization parameters that we use in this paper are listed in Appendix B.

\section{THE PARTICLE-WITHOUT-PARTICLE FORMULATION}

Before we introduce the multi-domain framework for the numerical calculations that we present in this paper, it is important first to look at its mathematical foundations. To that end, we only need to consider two subdomains of the physical domain, that is

$$
r^{*} \in(-\infty, \infty)=\left(-\infty, r_{p}^{*}\right) \cup\left(r_{p}^{*},+\infty\right),
$$

where $r_{p}^{*}=r_{p}^{*}(t)$ is the radial tortoise coordinate of the particle. This particular division obeys to the fact (see Paper I) that the key point of our technique consists in locating the particle between two subdomains, which automatically makes all the wave-type equations governing the dynamics of the scalar field modes to be homogeneous. As a direct consequence, we avoid all the problems associated with source terms with support at just one point and prevents us from introducing an artificial scale associated with the charged particle.

The problem is thus reduced to the junction conditions for the field across the particle. It is well-known (see, e.g. 65) that discontinuities in hyperbolic equations (like the wave equation) can only appear and propagate 
along characteristics. In order to analyze the junction conditions, it is therefore convenient to adopt a hyperbolic formulation of the equations for the scalar field. To that end, we introduce the variables

$$
\boldsymbol{U}=\left(\psi^{\ell m}, \phi^{\ell m}, \varphi^{\ell m}\right)=\left(r \Phi^{\ell m}, \partial_{t} \psi^{\ell m}, \partial_{r^{*}} \psi^{\ell m}\right) .
$$

at each of the two subdomains in (14), these satisfy the first-order system of PDEs (see [66] for an analysis in second-order form):

$$
\partial_{t} \boldsymbol{U}=\mathbb{A} \cdot \partial_{r^{*}} \boldsymbol{U}+\mathbb{B} \cdot \boldsymbol{U}+\boldsymbol{S},
$$

where the matrices $\mathbb{A}$ and $\mathbb{B}$ are given by

$$
\mathbb{A}=\left(\begin{array}{lll}
0 & 0 & 0 \\
0 & 0 & 1 \\
0 & 1 & 0
\end{array}\right), \quad \mathbb{B}=\left(\begin{array}{ccc}
0 & 1 & 0 \\
-V_{\ell} & 0 & 0 \\
0 & 0 & 0
\end{array}\right)
$$

and the vector $\boldsymbol{S}$ is

$$
\boldsymbol{S}=\left(0,-\frac{S^{\ell m}}{f\left(r_{p}\right)} \delta\left(r^{*}-r_{p}^{*}(t)\right), 0\right) .
$$

One can check that the system of PDEs given in Eq. (16) constitutes a first-order symmetric hyperbolic system. In order to analyze the jumps across the particle in terms of the variables of Eq. 15, we introduce the following splitting based on the domain division of Eq. (14):

$$
\begin{aligned}
\boldsymbol{U}\left(t, r^{*}\right) & =\boldsymbol{U}_{-}\left(t, r^{*}\right) \Theta\left(r_{p}^{*}(t)-r^{*}\right) \\
& +\boldsymbol{U}_{+}\left(t, r^{*}\right) \Theta\left(r^{*}-r_{p}^{*}(t)\right)
\end{aligned}
$$

where $\boldsymbol{U}_{-}$and $\boldsymbol{U}_{+}$are going to be smooth functions on $\left(-\infty, r_{p}^{*}\right)$ and on $\left(r_{p}^{*},+\infty\right)$ respectively, and $\Theta$ denotes the Heaviside step function. Introducing this ansazt [Eq. [19] ] into the system (16) and using the following definition of the jump in an arbitrary quantity $\lambda$

$$
[\lambda]_{p}=\lim _{r^{*} \rightarrow r_{p}^{*}} \lambda_{+}\left(t, r^{*}\right)-\lim _{r^{*} \rightarrow r_{p}^{*}} \lambda_{-}\left(t, r^{*}\right),
$$

we obtain the evolution equations for $\boldsymbol{U}_{ \pm}$:

$$
\partial_{t} \boldsymbol{U}_{ \pm}=\mathbb{A} \cdot \partial_{r^{*}} \boldsymbol{U}_{ \pm}+\mathbb{B} \cdot \boldsymbol{U}_{ \pm}
$$

and the expressions for the jumps in $\boldsymbol{U}$ across the particle (see also [66] and Paper I):

$$
\begin{aligned}
{\left[\psi^{\ell m}\right]_{p} } & =0 \\
{\left[\phi^{\ell m}\right]_{p} } & =-\frac{\dot{r}_{p}^{*} S^{\ell m}}{\left(1-\dot{r}_{p}^{* 2}\right) f_{p}}, \\
{\left[\varphi^{\ell m}\right]_{p} } & =\frac{S^{\ell m}}{\left(1-\dot{r}_{p}^{* 2}\right) f_{p}},
\end{aligned}
$$

where the dot denotes differentiation with respect to the time coordinate $t, f_{p}=f\left(r_{p}\right)$ and $S^{\ell m}$ is given in Eq. (10). As we can see, the field is continuous across the particle, and only the time and radial derivatives of the field have a jump, which depend on the particle's speed and satisfy the following advection-like equation:

$$
\left[\phi^{\ell m}-\dot{r}_{p}^{*} \varphi^{\ell m}\right]_{p}=0 .
$$

For $r_{p}^{*}=$ const., i.e. $\dot{r}_{p}^{*}=0$, we recover the jump conditions for the circular case studied in Paper I.

As mentioned above, the jump conditions 222)-24 have to be imposed on the characteristic fields because discontinues propagate along the characteristics. In our case, the characteristic fields are: $\psi^{\ell m}$ (whose characteristic surfaces are the spacelike surfaces $\{t=$ const. $\}$ ), $U^{\ell m}=\phi^{\ell m}-\varphi^{\ell m}$ (whose characteristic surfaces are the null surfaces $\left\{t-r^{*}=\right.$ const. $\left.\}\right)$, and $V^{\ell m}=\phi^{\ell m}+\varphi^{\ell m}$ (whose characteristic surfaces are the null surfaces $\{t+$ $r^{*}=$ const. $\}$ ). See an illustration of this in Figure 2.

An alternative description of the system, which we shall also use in this work, is to choose a set of variables associated with the characteristic fields. The obvious choice is

$$
\boldsymbol{N}=\left(\psi^{\ell m}, U^{\ell m}, V^{\ell m}\right)
$$

As before, we can apply the same splitting of Eq. (19) to these variables and follow the same procedure to obtain a set of equations for $\boldsymbol{N}_{ \pm}$:

$$
\partial_{t} \boldsymbol{N}_{ \pm}=\mathbb{C} \cdot \partial_{r^{*}} \boldsymbol{N}_{ \pm}+\mathbb{D} \cdot \boldsymbol{N}_{ \pm}
$$

where the matrices $\mathbb{C}$ and $\mathbb{D}$ are

$$
\mathbb{C}=\left(\begin{array}{ccc}
0 & 0 & 0 \\
0 & -1 & 0 \\
0 & 0 & 1
\end{array}\right), \quad \mathbb{D}=\left(\begin{array}{ccc}
0 & 1 / 2 & 1 / 2 \\
-V_{\ell} & 0 & 0 \\
-V_{\ell} & 0 & 0
\end{array}\right),
$$

and the junction condition of Eq. 22 and

$$
\begin{aligned}
{\left[U^{\ell m}\right]_{p} } & =-\frac{S^{\ell m}}{\left(1-\dot{r}_{p}^{*}\right) f_{p}} \\
{\left[V^{\ell m}\right]_{p} } & =\frac{\dot{r}_{p}^{*} S^{\ell m}}{\left(1+\dot{r}_{p}^{*}\right) f_{p}}
\end{aligned}
$$

We can now establish what we call the Particle without Particle $(\mathrm{PwP})$ formulation of the problem. By splitting the physical domain from the particle location as in Eq. (14) we can introduce in a natural way the splitting in the dynamical variables given by Eq. (19) (and the equivalent one for the characteristic variables $\boldsymbol{N})$. In this way, the restriction of the global variables to the subdomains to the left of the particle, $\left(-\infty, r_{p}^{*}\right)$, and to the right of the particle, $\left(r_{p}^{*},+\infty\right), \boldsymbol{U}_{+}\left(\boldsymbol{N}_{+}\right)$and $\boldsymbol{U}_{-}\left(\boldsymbol{N}_{-}\right)$respectively, satisfy homogeneous hyperbolic equations 21 [Eq. 27 for the characteristic variables]. This means that we have got rid of the distributional source terms, due to the particle, that appeared in the global equations (16). Then, the presence of the particle is introduced in the communication between the variables of different subdomains through the junction conditions (22)- 24) [Eqs. 22], 29, and (30) for the characteristic variables]. 


\section{A PSC METHOD IMPLEMENTATION OF THE PWP SCHEME}

We now introduce a multidomain computational method that implements the $\mathrm{PwP}$ scheme described in the previous section. To that end, we follow the discussion of Paper I, generalizing the methods introduced there to include the case of generic orbits, in which the particle is not at rest in the physical domain.

The first step is the truncation of the physical domain (as opposed to compactification). Instead of considering the domain (14) we consider the following domain: $\Omega=\left[r_{\mathrm{H}}^{*}, r_{\mathrm{I}}^{*}\right]$, where $r_{\mathrm{H}}^{*}$ corresponds to the truncation in the direction to the $\mathrm{MBH}$ horizon (at $r^{*} \rightarrow-\infty$ ) and $r_{\mathrm{I}}^{*}$ corresponds to the truncation in the direction to spatial infinity $\left(r^{*} \rightarrow+\infty\right)$. Next, we divide this truncated domain into a number $D$ of subdomains:

$$
\Omega=\bigcup_{\mathrm{A}=1}^{D} \Omega_{\mathrm{A}}, \quad \Omega_{\mathrm{A}}=\left[r_{\mathrm{A}, \mathrm{L}}^{*}, r_{\mathrm{A}, \mathrm{R}}^{*}\right],
$$

where $r_{\mathrm{A}, \mathrm{L}}^{*}$ and $r_{\mathrm{A}, \mathrm{R}}^{*}$ are the left and right boundaries of the subdomain $\Omega_{\mathrm{A}}$. Following the $\mathrm{PwP}$ scheme we place the particle at the interface between two of the subdomains. Now, let us assume that the particle is located at the interface between the subdomains $\Omega_{\mathrm{P}}$ and $\Omega_{\mathrm{P}+1}$, then

$$
r_{p}^{*}=r_{\mathrm{P}, \mathrm{R}}^{*}=r_{\mathrm{P}+1, \mathrm{~L}}^{*} .
$$

The main difference with respect to the circular case of Paper I is that now $r_{p}^{*}$ is a function of the time $t$. This implies that $r_{\mathrm{P}, \mathrm{R}}^{*}$ and $r_{\mathrm{P}+1, \mathrm{~L}}^{*}$ must be functions of $t$ as well, and the subdomains will in general change with time. We do not need that all subdomains change with time however, so we distinguish between dynamical and nondynamical subdomains according to whether or not any of their boundary nodes moves with time. This multidomain framework is illustrated in Fig. 1. In the remainder of this work we restrict ourselves to the case in which only the two subdomains that surround the particle are dynamical, i.e. only $r_{\mathrm{P}, \mathrm{R}}^{*}$ and $r_{\mathrm{P}+1, \mathrm{~L}}^{*}$ are time dependent.

The first ingredient in applying the PSC method is the discretization of the spatial domain (see 67] for a multidomain PSC method for elliptic equations). In our scheme, each subdomain is discretized independently, by using a Lobatto-Chebyshev grid (see Paper I for details on our particular implementation, and e.g. 68, for general details on the method), with collocation points given by

$$
X_{i}=-\cos \left(\frac{\pi i}{N}\right) \quad(i=0, \ldots, N) .
$$

In order to complete the discretization of the physical domain, we need to specify how the spectral coordinate $X$, associated with the Chebyshev polynomials, is related to the physical coordinate $r^{*}$. This is the key ingredient in our scheme; it is here where we establish how to keep the particle at the interface of two subdomains even

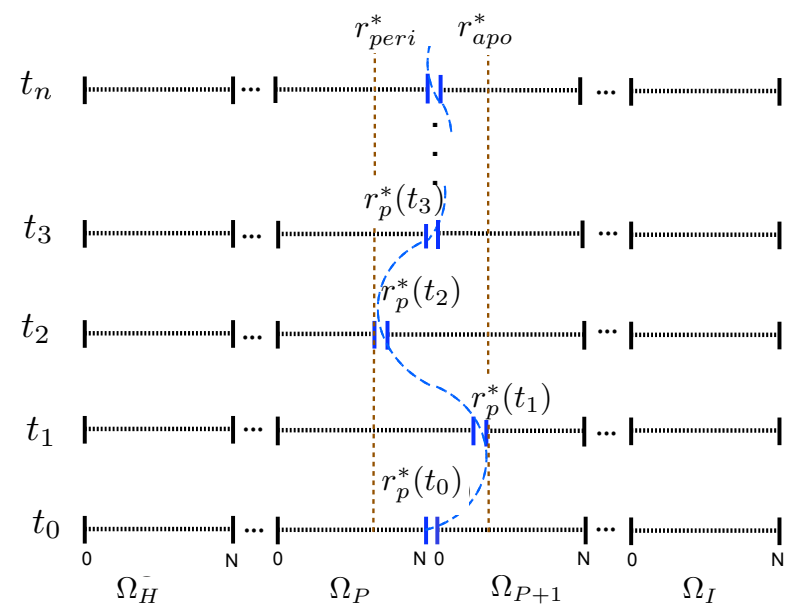

FIG. 1: The figure shows the structure of the one-dimensional spatial grid, the division in subdomains and the location of the particle at the interface between two of them, $r_{p}^{*}(t)$. In a generic orbit, the particle moves in a periodic way between the minimum (pericenter, denoted by $r_{\text {peri }}$ ) and the maximum (apocenter, denoted by $r_{\text {apo }}$ ). In this example, only the domains on the left and on the right of the particle are dynamical, i.e. they are the only ones whose coordinate size changes in time.

when it is moving in the radial direction. This information is encoded in the mapping from the spectral and physical domains. We have effectively traded particle motion for coordinate mappings. Given that the particle will be in general moving, it is convenient to prescribe a spacetime mapping. Since we discretize each such subdomain independently, and the spectral domains are always the interval $[-1,1]$, there are going to be as many mappings as subdomains. Then, for a given subdomain $\Omega_{\mathrm{A}}=\left[r_{\mathrm{A}, \mathrm{L}}^{*}, r_{\mathrm{A}, \mathrm{R}}^{*}\right](A=0, \ldots, D)$, the spacetime mapping to the spectral domain is taken to be linear and given by:

$$
\begin{aligned}
X_{\mathrm{A}}: \mathbb{T} \times\left[r_{\mathrm{A}, \mathrm{L}}^{*}, r_{\mathrm{A}, \mathrm{R}}^{*}\right] & \longrightarrow \mathbb{T} \times[-1,1] \\
\left(t, r^{*}\right) & \longmapsto\left(T, X_{\mathrm{A}}\right)
\end{aligned}
$$

where $\mathbb{T}=\left[t_{o}, t_{f}\right]$ is the evolution time interval and

$$
\begin{aligned}
T(t) & =t \\
X_{\mathrm{A}}\left(t, r^{*}\right) & =\frac{2 r^{*}-r_{\mathrm{A}, \mathrm{L}}^{*}-r_{\mathrm{A}, \mathrm{R}}^{*}}{r_{\mathrm{A}, \mathrm{R}}^{*}-r_{\mathrm{A}, \mathrm{L}}^{*}} .
\end{aligned}
$$

Here, the time dependence in $X_{\mathrm{A}}$ comes from the variation of $r_{\mathrm{A}, \mathrm{L}}^{*}$ or $r_{\mathrm{A}, \mathrm{R}}^{*}$ in time. The inverse (linear) mappings from the spectral domain to each of the subdomains $\Omega_{\mathrm{A}}$ are given by

$$
\begin{aligned}
\left.r^{*}\right|_{\Omega_{\mathrm{A}}}: \mathbb{T} \times[-1,1] & \longrightarrow \mathbb{T} \times\left[r_{\mathrm{A}, \mathrm{L}}^{*}, r_{\mathrm{A}, \mathrm{R}}^{*}\right] \\
(T, X) & \longmapsto\left(t, r^{*}\right)
\end{aligned}
$$


and

$$
\begin{aligned}
t(T) & =T \\
\left.r^{*}(T, X)\right|_{\Omega_{\mathrm{A}}} & =\frac{r_{\mathrm{A}, \mathrm{R}}^{*}-r_{\mathrm{A}, \mathrm{L}}^{*}}{2} X+\frac{r_{\mathrm{A}, \mathrm{L}}^{*}+r_{\mathrm{A}, \mathrm{R}}^{*}}{2} .
\end{aligned}
$$

In the PSC method we have two expansions (representations) of our variables: The spectral and the physical representations. The former expands the variables in a basis of Chebyshev polynomials, $\left\{T_{n}(X)\right\}(X \in[-1,1]$, $n=0, \ldots, N)$. Thus, our variables $\boldsymbol{U}$ can be approximated by

$$
\boldsymbol{U}_{N}\left(t, r^{*}\right)=\sum_{n=0}^{N} \boldsymbol{a}_{n}(t) T_{n}\left(X_{\mathrm{A}}\left(t, r^{*}\right)\right),
$$

where the $\boldsymbol{a}_{n}$ are the spectral coefficients which, in our case, will be time dependent. The physical representation approximates the solution as

$$
\boldsymbol{U}_{N}\left(t, r^{*}\right)=\sum_{i=0}^{N} \boldsymbol{U}_{i}(t) \mathcal{C}_{i}\left(X_{\mathrm{A}}\left(t, r^{*}\right)\right),
$$

where $\mathcal{C}_{i}(X)$ are the cardinal functions associated with our choice of collocation grid (Lobatto-Chebyshev) which satisfy the relation $\mathcal{C}_{i}\left(X_{j}\right)=\delta_{i j}$. In this representation, the coefficients $\left\{\boldsymbol{U}_{i}\right\}_{i=0, \ldots, N}$ are the values of our variables

$$
\boldsymbol{U}\left(t, r^{*}\left(T(t), X_{i}\right)\right) \equiv \boldsymbol{U}_{i}(t) \quad(i=0, \ldots, N),
$$

at the collocation points.

In these two representations we have two types of unknowns, the coefficients $\left\{\boldsymbol{a}_{n}\right\}_{n=0, \ldots, N}$ and the collocation values $\left\{\boldsymbol{U}_{i}\right\}_{i=0, \ldots, N}$. These $N+1$ unknowns are fully determined, at each subdomain, by imposing our set of PDEs (21) [or Eq. [27]] at each of the collocation points.

Then, the discretized version of the evolution equations 21) at each subdomain $\Omega_{\mathrm{A}}$ takes the following form:

$$
\partial_{T} \boldsymbol{U}_{i}=\mathbb{P}_{i}\left(\partial_{X} \boldsymbol{U}\right)_{i}+\mathbb{Q}_{i} \boldsymbol{U}_{i},
$$

where there is no summation over $i$ and the matrices $\mathbb{P}_{i}$ and $\mathbb{Q}_{i}$ are given by

$$
\begin{aligned}
\mathbb{P}_{i} & =\left(\begin{array}{ccc}
0 & 0 & 0 \\
0 & -\left(\partial_{t} X_{\mathrm{A}}\right)_{i} & \left(\partial_{r^{*}} X_{\mathrm{A}}\right)_{i} \\
0 & \left(\partial_{r^{*}} X_{\mathrm{A}}\right)_{i} & -\left(\partial_{t} X_{\mathrm{A}}\right)_{i}
\end{array}\right) \\
\mathbb{Q}_{i} & =\left(\begin{array}{ccc}
0 & 1 & -\frac{\left(\partial_{t} X_{\mathrm{A}}\right)_{i}}{\left(\partial_{r^{*}} X_{\mathrm{A}}\right)_{i}} \\
-V_{\ell}^{\mathrm{A}, i} & 0 & 0 \\
0 & 0 & 0
\end{array}\right)
\end{aligned}
$$

the quantity $V_{\ell}^{\mathrm{A}, i}$ is given by

$$
V_{\ell}^{\mathrm{A}, i}(t)=V_{\ell}\left(r^{*}\left(T(t), X_{\mathrm{A}, i}\right)\right),
$$

$X_{\mathrm{A}, i}$ is the $i$-th collocation point at the domain $\Omega_{\mathrm{A}}$, given by Eq. (33), and

$$
\begin{gathered}
\left(\partial_{r^{*}} X_{\mathrm{A}}\right)_{i}=\frac{2}{r_{\mathrm{A}, \mathrm{R}}^{*}-r_{\mathrm{A}, \mathrm{L}}^{*}} . \\
\left(\partial_{t} X_{\mathrm{A}}\right)_{i}=-\frac{\dot{r}_{p}^{*}}{r_{\mathrm{A}, \mathrm{R}}^{*}-r_{\mathrm{A}, \mathrm{L}}^{*}}\left\{\delta_{p}^{\mathrm{A}, \mathrm{L}}+\delta_{p}^{\mathrm{A}, \mathrm{R}}+\left(\delta_{p}^{\mathrm{A}, \mathrm{R}}-\delta_{p}^{\mathrm{A}, \mathrm{L}}\right) X_{\mathrm{A}, i}\right\}, \\
\delta_{p}^{\mathrm{A}, \mathrm{L}}=\left\{\begin{array}{ll}
1 & \text { if } r_{p}^{*}=r_{\mathrm{A}, \mathrm{L}}^{*}, \\
0 & \text { otherwise }
\end{array} \quad \delta_{p}^{\mathrm{A}, \mathrm{R}}= \begin{cases}1 & \text { if } r_{p}^{*}=r_{\mathrm{A}, \mathrm{R}}^{*}, \\
0 & \text { otherwise. }\end{cases} \right.
\end{gathered}
$$

For a given set of initial data

$$
\boldsymbol{U}_{i}^{o}=\boldsymbol{U}\left(t=t_{o}, r^{*}\left(T, X_{\mathrm{A}, i}\right)\right),
$$

the system of $N+1$ ODEs defined by Eq. (41) determines uniquely the values of our variables $\boldsymbol{U}$ at the collocation points, i.e. the $N+1$ unknowns, which in turn determines uniquely our approximation to the solution via Eq. (39). Looking at the expressions for the matrices $\mathbb{P}_{i}$ and $\mathbb{Q}_{i}$ [Eqs. 42 and $[43$ ] we see that their components are explicitly time dependent in the case of a dynamical subdomain (in the case of this paper, only those that surround the particle).

A similar system of equations can be obtained for the characteristic variables $\boldsymbol{N}$ [Eq. 26]],

$$
\partial_{T} \boldsymbol{N}_{i}=\mathbb{R}_{i}\left(\partial_{X} \boldsymbol{N}\right)_{i}+\mathbb{S}_{i} \boldsymbol{N}_{i}
$$

where again there is no summation over $i$ and the matrices $\mathbb{R}_{i}$ and $\mathbb{S}_{i}$ are

$$
\begin{aligned}
\mathbb{R}_{i} & =\left(\begin{array}{ccc}
0 & 0 & 0 \\
0 & -\left(\partial_{t} X_{\mathrm{A}}\right)_{i}-\left(\partial_{r^{*}} X_{\mathrm{A}}\right)_{i} & 0 \\
0 & 0 & -\left(\partial_{t} X_{\mathrm{A}}\right)_{i}+\left(\partial_{r^{*}} X_{\mathrm{A}}\right)_{i}
\end{array}\right) \\
\mathbb{S}_{i} & =\left(\begin{array}{ccc}
0 & \frac{1}{2}\left[1+\frac{\left(\partial_{t} X_{\mathrm{A}}\right)_{i}}{\left(\partial_{r^{*}} X_{\mathrm{A}}\right)_{i}}\right] & \frac{1}{2}\left[1-\frac{\left(\partial_{t} X_{\mathrm{A}}\right)_{i}}{\left(\partial_{r^{*}} X_{\mathrm{A}}\right)_{i}}\right] \\
-V_{\ell}^{\mathrm{A}, i} & 0 & 0 \\
-V_{\ell}^{\mathrm{A}, i} & 0 & 0
\end{array}\right)(51)
\end{aligned}
$$

However, the ODEs given in Eq. (41) do not constitute a complete description of our system. We still need to incorporate all the necessary boundary conditions to adequately describe the system. First of all, we have the global boundary conditions, at $r_{\mathrm{H}}^{*}$ and $r_{\mathrm{I}}^{*}$ to prevent incoming signals from outside the physical domain, $\Omega=\left[r_{\mathrm{H}}^{*}, r_{\mathrm{I}}^{*}\right]$, sometimes known as absorbing boundary conditions. In this work we try to push the boundaries as far as possible (typically in such a way that, given the evolution time, $t_{f}-t_{o}$, the boundaries are not in causal contact with the particle) and impose Sommerfeld outgoing boundary conditions

$$
\begin{aligned}
& \phi^{\ell m}\left(t, r_{\mathrm{H}}^{*}\right)-\varphi^{\ell m}\left(t, r_{\mathrm{H}}^{*}\right)=0, \\
& \phi^{\ell m}\left(t, r_{\mathrm{I}}^{*}\right)+\varphi^{\ell m}\left(t, r_{\mathrm{I}}^{*}\right)=0 .
\end{aligned}
$$


These are exact boundary conditions only when $V_{\ell}=0$ or $r_{\mathrm{H}}^{*} \rightarrow-\infty$ and $r_{\mathrm{I}}^{*} \rightarrow+\infty$.

The second type of boundary conditions that need to be imposed are the junction conditions between the different subdomains. In this paper we present two different methods of imposing them: (i) The penalty method, which has already been used in Paper I, and (ii) direct communication of the characteristic fields. In the remainder of this section we describe both methods and the modifications that have to be included for their implementation in the system of ODEs (41). We recall that in this paper we consider the case in which the only dynamical subdomains are those two surrounding the particle, $\Omega_{\mathrm{P}}$ and $\Omega_{\mathrm{P}+1}$. In this way the particle is located at their interface as described by Eq. (32). We also have: $r_{\mathrm{A}, \mathrm{R}}^{*}=r_{\mathrm{A}, \mathrm{N}}^{*}$ and $r_{\mathrm{A}+1, \mathrm{~L}}^{*}=r_{\mathrm{A}+1,0}^{*}$.

\section{A. The Penalty method}

The basic idea behind the penalty method is to modify the equations by adding terms that dynamically drive the system to a state in which the junction conditions are satisfied to a high degree of precision. These additional terms are proportional to the conditions that one is interesting in imposing. In our case they are proportional to the junction conditions. Details of the method can be found in Paper I (for generalities see, e.g. [69]).

An important point in using the penalty method is that since we are dealing with hyperbolic equations, the junction conditions have to be imposed across the characteristics (see Figure 2), and this determines which combinations of the junction conditions have to be used in each equation.

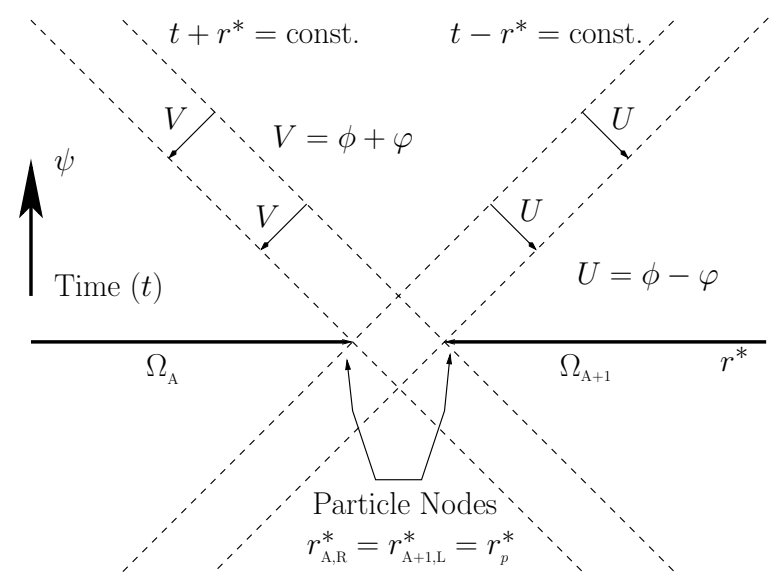

FIG. 2: Characteristic structure of our field equations. The picture shows the characteristics $\left(t \pm r^{*}=\right.$ const.) of the hyperbolic equations that describe the system [Eq. (16)], the characteristic fields $\left(\psi^{\ell m}, U^{\ell m}=\phi^{\ell m}-\varphi^{\ell m}\right.$, and $V^{\ell m}=$ $\left.\phi^{\ell m}+\varphi^{\ell m}\right)$, and their propagation direction.

In what follows we list the different equations for the different cases that appear depending on whether we are dealing with a subdomain to the left or to the right of the particle, and also on whether the variables are associated with boundary points or not. That is, we have four basic cases (other possibilities can be derived from these cases): (i) Inner points for the subdomain to the left of the particle, i.e. $\Omega_{\mathrm{P}}$; (ii) inner points for a subdomain to the right of the particle, i.e. $\Omega_{\mathrm{P}+1}$; (iii) left boundary point; and (iv) right boundary point.

(i) The equations for inner points $(i=1, \ldots, N-1)$ valid at the dynamical subdomain at the left of the particle (to simplify the notation we have dropped the harmonic indices $\ell$ and $m$ ) are:

$$
\begin{aligned}
\partial_{T} \psi_{\mathrm{P}, i} & =\dot{r}_{p}^{*} \frac{1+X_{\mathrm{P}, i}}{2} \varphi_{\mathrm{P}, i}+\phi_{\mathrm{P}, i}, \\
\partial_{T} \phi_{\mathrm{P}, i} & =\dot{r}_{p}^{*} \frac{1+X_{\mathrm{P}, i}}{2}\left(\partial_{r^{*}} \phi_{\mathrm{P}}\right)_{i}+\left(\partial_{r^{*}} \varphi_{\mathrm{P}}\right)_{i} \\
& -V_{\ell}^{\mathrm{P}, i} \psi_{\mathrm{P}, i}, \\
\partial_{T} \varphi_{\mathrm{P}, i} & =\dot{r}_{p}^{*} \frac{1+X_{\mathrm{P}, i}}{2}\left(\partial_{r^{*}} \varphi_{\mathrm{P}}\right)_{i}+\left(\partial_{r^{*}} \phi_{\mathrm{P}}\right)_{i},
\end{aligned}
$$

where for any quantity $\mathcal{A}$ we compute the spatial derivatives as

$$
\left(\partial_{r^{*}} \mathcal{A}_{\mathrm{P}}\right)_{i}=\left(\partial_{r^{*}} X_{\mathrm{P}}\right)_{i}\left(\partial_{X} \mathcal{A}_{\mathrm{P}}\right)_{i},
$$

and $\left(\partial_{r^{*}} X_{\mathrm{P}}\right)_{i}$ is given in Eq. 45.

(ii) Equations for inner points at the dynamical subdomain at the right of the particle:

$$
\begin{aligned}
\partial_{T} \psi_{\mathrm{P}+1, i} & =\dot{r}_{p}^{*} \frac{1-X_{\mathrm{P}+1, i}}{2} \varphi_{\mathrm{P}+1, i}+\phi_{\mathrm{P}+1, i} \\
\partial_{T} \phi_{\mathrm{P}+1, i} & =\dot{r}_{p}^{*} \frac{1-X_{\mathrm{P}+1, i}}{2}\left(\partial_{r^{*}} \phi_{\mathrm{P}+1}\right)_{i}+\left(\partial_{r^{*}} \varphi_{\mathrm{P}+1}\right)_{i} \\
& -V_{\ell}^{\mathrm{P}+1, i} \psi_{\mathrm{P}+1, i} \\
\partial_{T} \varphi_{\mathrm{P}+1, i} & \left.=\dot{r}_{p}^{*} \frac{1-X_{\mathrm{P}+1, i}}{2}\left(\partial_{r^{*}} \varphi_{\mathrm{P}+1}\right)_{i}+\left(\partial_{r^{*}} \phi_{\mathrm{P}+1}\right)_{i} 60\right)
\end{aligned}
$$

The equations at the inner points of any non-dynamical subdomain are obtained by setting $\dot{r}_{p}^{*}=0$ either at the set of equations (54)-(56) or at the set of equations (58)60).

(iii) Equations valid at the right boundary of the dynamical subdomain at the left of the particle, that is, at $r^{*}=r_{\mathrm{P}, \mathrm{N}}^{*}=r_{p}^{*}(t)$ :

$$
\begin{aligned}
\partial_{T} \psi_{\mathrm{P}, \mathrm{N}} & =\dot{r}_{p}^{*} \varphi_{\mathrm{P}, \mathrm{N}}+\phi_{\mathrm{P}, \mathrm{N}} \\
& -\tau_{\psi}^{\mathrm{P}, \mathrm{R}}\left(\psi_{\mathrm{P}, \mathrm{N}}-\psi_{\mathrm{P}+1,0}\right), \\
\partial_{T} \phi_{\mathrm{P}, \mathrm{N}} & =\dot{r}_{p}^{*}\left(\partial_{r^{*}} \phi_{\mathrm{P}}\right)_{\mathrm{N}}+\left(\partial_{r^{*}} \varphi_{\mathrm{P}}\right)_{\mathrm{N}}-V_{\ell}^{\mathrm{P}, \mathrm{N}} \psi_{\mathrm{P}, \mathrm{N}} \\
& -\frac{\tau_{\phi}^{\mathrm{P}, \mathrm{R}}}{2}\left[\phi_{\mathrm{P}, \mathrm{N}}+\varphi_{\mathrm{P}, \mathrm{N}}-\left(\phi_{\mathrm{P}+1,0}+\varphi_{\mathrm{P}+1,0}\right)\right. \\
& \left.+[\phi]_{p}^{\mathrm{P}, \mathrm{P}+1}+[\varphi]_{p}^{\mathrm{P} \mathrm{P}+1}\right], \\
\partial_{T} \varphi_{\mathrm{P}, \mathrm{N}} & =\dot{r}_{p}^{*}\left(\partial_{r^{*}} \varphi_{\mathrm{P}}\right)_{\mathrm{N}}+\left(\partial_{r^{*}} \phi_{\mathrm{P}}\right)_{\mathrm{N}} \\
& -\frac{\tau_{\varphi}^{\mathrm{P}, \mathrm{R}}}{2}\left[\phi_{\mathrm{P}, \mathrm{N}}+\varphi_{\mathrm{P}, \mathrm{N}}-\left(\phi_{\mathrm{P}+1,0}+\varphi_{\mathrm{P}+1,0}\right)\right. \\
& \left.+[\phi]_{p}^{\mathrm{P}, \mathrm{P}+1}+[\varphi]_{p}^{\mathrm{P}, \mathrm{P}+1}\right],
\end{aligned}
$$


where $\tau_{\psi}^{\mathrm{P}, \mathrm{R}}, \tau_{\phi}^{\mathrm{P}, \mathrm{R}}$, and $\tau_{\varphi}^{\mathrm{P}, \mathrm{R}}$, are penalty (constant) coefficients associated with the evolution of $\psi_{\mathrm{P}, \mathrm{N}}(T), \phi_{\mathrm{P}, \mathrm{N}}(T)$, and $\varphi_{\mathrm{P}, \mathrm{N}}(T)$ respectively. The quantities $[\phi]_{p}^{\mathrm{P}, \mathrm{P}+1}$ and $[\varphi]_{p}^{\mathrm{P}, \mathrm{P}+1}$ are the jumps across the subdomains $\Omega_{\mathrm{P}}$ and $\Omega_{\mathrm{P}+1}$, given in Eqs. (23) and (24).

(iv) Equations valid at the left boundary of the dynamical subdomain at the right of the particle, i.e. at $r^{*}=r_{\mathrm{P}+1,0}^{*}=r_{p}^{*}(t)$ :

$$
\begin{aligned}
\partial_{T} \psi_{\mathrm{P}+1,0} & =\dot{r}_{p}^{*} \varphi_{\mathrm{P}+1,0}+\phi_{\mathrm{P}+1,0} \\
& -\tau_{\psi}^{\mathrm{P}+1, \mathrm{~L}}\left(\psi_{\mathrm{P}+1,0}-\psi_{\mathrm{P}, \mathrm{N}}\right), \\
\partial_{T} \phi_{\mathrm{P}+1,0} & =\dot{r}_{p}^{*}\left(\partial_{r^{*}} \phi_{\mathrm{P}+1}\right)_{0}+\left(\partial_{r^{*}} \varphi_{\mathrm{P}+1}\right)_{0}-V_{\ell}^{\mathrm{P}+1,0} \psi_{\mathrm{P}+1,0} \\
& -\frac{\tau_{\phi}^{\mathrm{P}+1, \mathrm{~L}}}{2}\left[\phi_{\mathrm{P}+1,0}-\varphi_{\mathrm{P}+1,0}-\left(\phi_{\mathrm{P}, \mathrm{N}}-\varphi_{\mathrm{P}, \mathrm{N}}\right)\right. \\
& \left.-[\phi]_{p}^{\mathrm{P}, \mathrm{P}+1}+[\varphi]_{p}^{\mathrm{P}, \mathrm{P}+1}\right], \\
\partial_{T} \varphi_{\mathrm{P}+1,0} & =\dot{r}_{p}^{*}\left(\partial_{r^{*}} \varphi_{\mathrm{P}+1}\right)_{0}+\left(\partial_{r^{*}} \phi_{\mathrm{P}+1}\right)_{0} \\
& +\frac{\tau_{\varphi}^{\mathrm{P}+1, \mathrm{~L}}}{2}\left[\phi_{\mathrm{P}+1,0}-\varphi_{\mathrm{P}+1,0}-\left(\phi_{\mathrm{P}, \mathrm{N}}-\varphi_{\mathrm{P}, \mathrm{N}}\right)\right. \\
& \left.-[\phi]_{p}^{\mathrm{P}, \mathrm{P}+1}+[\varphi]_{p}^{\mathrm{P}, \mathrm{P}+1}\right],
\end{aligned}
$$

where again $\tau_{\psi}^{\mathrm{P}+1, \mathrm{~L}}, \tau_{\phi}^{\mathrm{P}+1, \mathrm{~L}}$, and $\tau_{\varphi}^{\mathrm{P}+1, \mathrm{~L}}$ are penalty coefficients associated with the evolution of $\psi_{\mathrm{P}+1,0}(T)$, $\phi_{\mathrm{P}+1,0}(T)$, and $\varphi_{\mathrm{P}+1,0}(T)$ respectively. At this point we comment on the equation for $\psi^{\ell m}$. Here, we are enforcing the continuity of this variable via a penalty term. However, as we already mentioned in Paper I, there is an alternative to this, which consists in taking a sort of average of the right-hand side of the equation for $\psi^{\ell m}$ over the two subdomains that we are trying to communicate or match. The alternative equation for $\psi^{\ell m}$ would look as follows:

$$
\partial_{T} \psi_{\mathrm{P}+1,0}=\frac{1}{2} \dot{r}_{p}^{*}\left(\varphi_{\mathrm{P}, \mathrm{N}}+\varphi_{\mathrm{P}+1,0}\right)+\frac{1}{2}\left(\phi_{\mathrm{P}, \mathrm{N}}+\phi_{\mathrm{P}+1,0}\right),
$$

and the same applies to the equation for $\psi_{\mathrm{P}, 0}$. In this way, $\psi$ is continuous, to machine precision, by construction.

Finally, the outgoing (global) boundary conditions described by Eqs. (52) and (53) are equivalent to the following equations: At the horizon $r^{*}=r_{\mathrm{H}}^{*}$ :

$$
\begin{aligned}
\partial_{T} \psi_{1, \mathrm{H}} & =\phi_{1, \mathrm{H}}, \\
\partial_{T} \phi_{1, \mathrm{H}} & =\partial_{T} \varphi_{1, \mathrm{H}}, \\
\partial_{T} \varphi_{1, \mathrm{H}} & =\left(\partial_{r^{*}} \phi_{1}\right)_{\mathrm{H}},
\end{aligned}
$$

and at spatial infinity $r^{*}=r_{\mathrm{I}}^{*}$ :

$$
\begin{aligned}
\partial_{T} \psi_{\mathrm{D}, \mathrm{I}} & =\phi_{\mathrm{D}, \mathrm{I}}, \\
\partial_{T} \phi_{\mathrm{D}, \mathrm{I}} & =-\partial_{T} \varphi_{\mathrm{D}, \mathrm{I}}, \\
\partial_{T} \varphi_{\mathrm{D}, \mathrm{I}} & =\left(\partial_{r^{*}} \phi_{\mathrm{D}}\right)_{\mathrm{I}},
\end{aligned}
$$

where the subindex 1 refers to the first subdomain and $D$ to the last one.

\section{B. Evolution of the characteristic fields}

An alternative to the penalty method is to stick to hyperbolic methods and use the symmetric hyperbolic structure of our system in order to communicate the different subdomains by passing the characteristic fields through the corresponding characteristics. This process can be illustrated with the use of Figure 2, Let us consider the interface between two arbitrary subdomains, say $\Omega_{\mathrm{A}}$ and $\Omega_{\mathrm{A}+1}$. The boundary nodes are $r_{\mathrm{A}, \mathrm{R}}^{*}=r_{\mathrm{A}, \mathrm{N}}^{*}$ and $r_{\mathrm{A}+1, \mathrm{~L}}^{*}=r_{\mathrm{A}+1,0}^{*}$. Then, looking at the representation of the propagation of the characteristic fields, $U^{\ell m}$ and $V^{\ell, m}$ in Figure 2, we can deduce that at $r^{*}=r_{\mathrm{A}, \mathrm{R}}^{*}$ we only need to evolve Eqs. 49 for $\left(\psi_{\mathrm{A}, \mathrm{N}}, U_{\mathrm{A}, \mathrm{N}}\right)$, whereas $V_{\mathrm{A}, \mathrm{N}}$ is just obtained by copying the value of $V_{\mathrm{A}+1,0}$ from the other subdomain. In contrast, at $r^{*}=r_{\mathrm{A}+1, \mathrm{~L}}^{*}$ we only need to evolve Eqs. 49 for $\left(\psi_{\mathrm{A}+1,0}, V_{\mathrm{A}+1,0}\right)$, whereas $Y_{\mathrm{A}+1,0}$ is obtained by copying the value of $U_{\mathrm{A}, \mathrm{N}}$ from the other subdomain. In the case where the particle is at the interface of these two subdomains (and hence they are dynamical subdomains), i.e. $A=P$, we cannot just copy the values of the characteristic fields, but instead we have to enforce the junction conditions of Eqs. 29) and (30). That is, we obtain $V_{\mathrm{P}, \mathrm{N}}$ by doing:

$$
V_{\mathrm{P}, \mathrm{N}}=V_{\mathrm{P}+1,0}-[V]_{p}^{\mathrm{P}, \mathrm{P}+1},
$$

and $U_{\mathrm{P}+1,0}$ by

$$
U_{\mathrm{P}+1,0}=U_{\mathrm{P}, \mathrm{N}}+[U]_{p}^{\mathrm{P}, \mathrm{P}+1} .
$$

The practical implementation of this technique requires to treat the different values of the fields at the different collocation points in a particular order. In what follows we outline an algorithm to do so and the associated equations. Step by step, the procedure is the following: (i) For each subdomain $\Omega_{\mathrm{A}}(A=1, \ldots, D)$, we evolve the variable $U^{\ell m}$ at the inner points and at the right boundary node, $r^{*}=r_{\mathrm{A}, \mathrm{N}}^{*}$, according to the following evolution equation (again we drop the harmonic indices $\ell$ and $m$ ):

$$
\partial_{T} U_{\mathrm{A}, i}=-\left[1+\frac{\left(\partial_{t} X_{\mathrm{A}}\right)_{i}}{\left(\partial_{r^{*}} X_{\mathrm{A}}\right)_{i}}\right]\left(\partial_{r^{*}} U_{\mathrm{A}}\right)_{i}-V_{\ell}^{\mathrm{A}, i} \psi_{\mathrm{A}, i},
$$

where $\left(\partial_{r^{*}} X_{\mathrm{A}}\right)_{i}$ and $\left(\partial_{t} X_{\mathrm{A}}\right)_{i}$ are given in Eqs. 45. and (46) respectively, and $i=1, \ldots, N$. The next step is: (ii) For each subdomain $\Omega_{\mathrm{A}}(A=1, \ldots, D)$, we evolve the variable $V^{\ell m}$ at the inner points and at the left boundary node, $r^{*}=r_{\mathrm{A}, 0}^{*}$, according to the following evolution equation:

$$
\partial_{T} V_{\mathrm{A}, i}=\left[1-\frac{\left(\partial_{t} X_{\mathrm{A}}\right)_{i}}{\left(\partial_{r^{*}} X_{\mathrm{A}}\right)_{i}}\right]\left(\partial_{r^{*}} V_{\mathrm{A}}\right)_{i}-V_{\ell}^{\mathrm{A}, i} \psi_{\mathrm{A}, i},
$$

where $i=0, \ldots, N-1$. (iii) We evolve the variable $V^{\ell m}$ at all the right boundary nodes according to Eq. (75). For the domains without the particle we just simply pass the value to the right. Here, we need to make an important 
remark about the practical implementation (in a numerical code) of this step. For the evolution of the timedependent variables of our system of ODEs, $\boldsymbol{N}_{\mathrm{A}, i}(T)$, we use two different methods. One implements directly Eq. (75). The other one, uses the method of lines (see, e.g. [70]), so that it incorporates the boundary matching conditions into the evaluation of the right-hand side of the evolution equations. Then, to insert this step into the method of lines of our numerical algorithm we need to use the derivative of Eq. 75 rather than this equation itself. That is, we need to use:

$$
\frac{d V_{\mathrm{P}, \mathrm{N}}}{d T}=\frac{d V_{\mathrm{P}+1,0}}{d T}-\frac{d[V]_{p}^{\mathrm{P}, \mathrm{P}+1}}{d T} .
$$

This means that we also need the expressions for the derivatives of the jumps in the variables $U^{\ell m}$ and $V^{\ell m}$. They can be directly derived from the expressions of the jumps [Eqs. 29) and [30] ] and the geodesic equations (see Appendix A). We give the resulting expressions in Appendix $\mathrm{C}$ in particular $d[V]_{p}^{\mathrm{P}, \mathrm{P}+1} / d T$ is given in Eq. (C2).

(iv) We evolve the variable $U^{\ell m}$ at all the left boundary nodes according to Eq. (76). Again, for domains that do not have the particle, we just pass the value of this variable, to the left in this case. In order to use the method of lines, following the previous discussion, we need to use the derivative of Eq. (76), that is,

$$
\frac{d U_{\mathrm{P}+1,0}}{d T}=\frac{d U_{\mathrm{P}, \mathrm{N}}}{d T}+\frac{d[U]_{p}^{\mathrm{P}, \mathrm{P}+1}}{d T},
$$

where $d[U]_{p}^{\mathrm{P}, \mathrm{P}+1} / d T$ is given in Eq. $\mathrm{C} 1$.

(v) We evolve the characteristic variables $U^{\ell m}$ and $V^{\ell m}$ at the global boundaries. At the horizon, $r^{*}=r_{\mathrm{H}}^{*}$, based on the method of lines, we use the equations:

$$
\begin{aligned}
& \partial_{T} U_{0, \mathrm{H}}=0, \\
& \partial_{T} V_{0, \mathrm{H}}=\left(\partial_{r^{*}} V_{0}\right)_{\mathrm{H}}-V_{\ell}^{0, \mathrm{H}} \psi_{0, \mathrm{H}} .
\end{aligned}
$$

Then, at spatial infinity $r^{*}=r_{\mathrm{I}}^{*}$ :

$$
\begin{aligned}
\partial_{T} U_{\mathrm{D}, \mathrm{I}} & =-\left(\partial_{r^{*}} U_{\mathrm{D}}\right)_{\mathrm{I}}-V_{\ell}^{\mathrm{D}, \mathrm{I}} \psi_{\mathrm{D}, \mathrm{I}}, \\
\partial_{T} V_{\mathrm{D}, \mathrm{I}} & =0 .
\end{aligned}
$$

(vi) The last step consists in evolving the variable $\psi^{\ell m}$ at the collocation points of all domains according to the equation

$$
\partial_{T} \psi_{\mathrm{A}, i}=\frac{1}{2}\left(U_{\mathrm{A}, i}+V_{\mathrm{A}, i}\right)+\frac{1}{2} \frac{\left(\partial_{t} X_{\mathrm{A}}\right)_{i}}{\left(\partial_{r^{*}} X_{\mathrm{A}}\right)_{i}}\left(U_{\mathrm{A}, i}-V_{\mathrm{A}, i}\right),
$$

where $i=0, \ldots, N$, and $A=1, \ldots, D$.

\section{RESULTS FROM THE SIMULATIONS}

In this section, we present the main results from the actual numerical calculations. These calculations have been done using implementations of the $\mathrm{PwP}$ formulation in combination with the multidomain scheme described above. The details of the numerical codes that we have written for the simulations are essentially the same as in the case of Paper I, so we refer the reader to this paper for the computational details. Here, we focus on the new aspects of the implementation.

The results that we present refer to three different types of eccentric orbits (see Paper I for the case of circular orbits). These three types of orbits, in terms of the eccentricity and semilatus rectum, are: (i) $(e, p)$ $=(0.1,6.3)$; (ii) $(e, p)=(0.3,6.7)$; and (iii) $(e, p)=$ $(0.5,7.1)$. We show pieces of these orbits in Figure 3 .

Before introducing the results, it is worth making a comment on the numerical implementation of the methods and techniques that we described above. In contrast with the work done for Paper I, where we only used the penalty method to communicate the subdomains, here we have implemented in our numerical codes, two different techniques: The penalty method, described in Sec. IVA, and the direct communication of the characteristic fields, described in Sec. IVB. In practice, the numerical implementation of the two methods is equivalent to having two different numerical codes for the evolution of the system. For the results presented in the paper we have mainly used the penalty formulation. But we have also used the characteristic one, the one based on direct communication of the characteristic fields, both to check the results, and for the numerical evaluation of the self-force below. A last comment on the implementation refers to the characteristic formulation. For the time evolution, like in Paper I, we use a Runge-Kutta 4 (RK4) algorithm (see, e.g. [71, 72]). Then, as we already discussed in Sec IVB, there are two ways of communicating the fields: Either we use the expressions containing the jumps in $\left(U^{\ell m}, V^{\ell m}\right)$, Eqs. 76$)$ and 75 , which requires some engineering in the RK4 algorithm, or we use the expressions containing the derivatives of the jumps in $\left(U^{\ell m}, V^{\ell m}\right)$, Eqs. 76$)$ and 75 , which can be adapted naturally with the method of lines. This second option requires to impose initially the values of the jumps, Eqs. (C1) and (C2), since during the evolution the only input about them is the information on their derivatives. Therefore, in this case, we cannot just use the zero initial data that we used in Paper I, and that can be used for the other cases presented here, namely $\boldsymbol{U}_{o}=\boldsymbol{U}\left(t=t_{o}, r\right)$ $=\left(\psi^{\ell m}\left(t_{o}, r\right), \phi^{\ell m}\left(t_{o}, r\right), \varphi^{\ell m}\left(t_{o}, r\right)\right)=\mathbf{0}$, but a modification of it, at least at the nodes where the particle is located. All these options have been implemented in our numerical codes.

The first results that we present refer to the validation of the code. In Paper I we already presented several tests. Here we show a multiple convergence plot (for the different orbits) that shows that our PwP scheme indeed provide solutions smooth enough to preserve the a priori exponential convergence of the PSC method. In Figure 4 we have plots for the evolution of the harmonic mode $(\ell, m)=(2,2)$, and the different orbits considered in 
Orbits around the $\mathrm{MBH}$
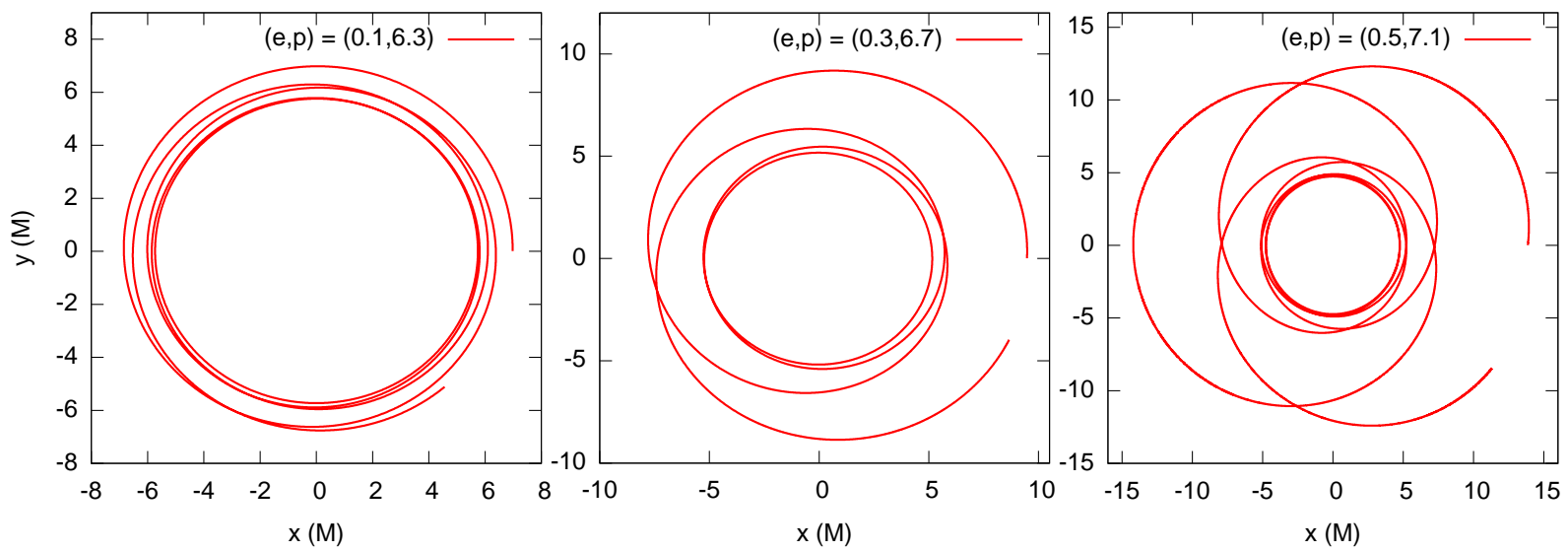

FIG. 3: Eccentric orbits considered in the numerical evolutions presented in this paper. From left to right we have: $(\mathrm{i})(e, p)=$ $(0.1,6.3)$; (ii) $(e, p)=(0.3,6.7)$; and (iii) $(e, p)=(0.5,7.1)$. These orbits have been integrated using the equations of Appendix A in particular Eqs. A5 and A6.

Figure 3 showing an estimation of the truncation error, $\log _{10}\left|a_{N}\right|$ [where the $a_{N}$ is the last spectral coefficient of the spectral approximation of Eq. [38] ], with respect to the number of collocation points, $N$. For these plots we use the information coming from the subdomain to the right of the particle, i.e. $\Omega_{\mathrm{P}+1}$, so that the particle is located at the left boundary node of this subdomain. As one can see, the truncation error, estimated as the absolute value of the last spectral coefficient, $\left|a_{N}\right|$, decreases exponentially with the number of collocation points, as expected in the PSC method for smooth solutions.

The key ingredient of our $\mathrm{PwP}$ scheme is to put the particle at the interface between two subdomains, so that it does not appear explicitly as a distributional term in the field equations, but as a boundary condition between the solution at adjoining subdomains. This provides smooth numerical solutions that satisfy the junction conditions dictated by the jumps [see Eqs. (23) and (24) or Eqs. [29) and [30]. We have performed simulations that use zero initial data (or initial data that is zero everywhere except at the particle nodes), that is

$$
\psi^{\ell m}\left(t_{o}, r^{*}\right)=\phi^{\ell m}\left(t_{o}, r^{*}\right)=\varphi^{\ell m}\left(t_{o}, r^{*}\right)=0 .
$$

As a consequence of this, the numerical evolution produces an initial unphysical burst. We have to wait until this unphysical feature propagates away in order to analyze the solution and obtain physically relevant results. In Figure 5 we show snapshots of the evolution of the $(\ell, m)=(2,2)$ harmonic mode, including details near the particle location, that illustrate the ability of our method to capture the structure of the solution near the particle, in particular the ability of resolving the jumps in the time and radial derivative of the field variables.

The main goal of this paper is the computation of the self-force, hence the last two plots that we present show results on the estimation of the regular field. From the values of the components of the gradient of the regular field, $\nabla_{\alpha} \Phi^{\mathrm{R}}$, the self-force can be directly computed by using Eq. (6). We have carried out evolutions of the orbits shown in Figure 3 and we have computed the values of the components of the gradient of the regular field along the evolution. This means that we have evolved all the necessary modes of the retarded field up to a maximum $\ell$, say $\ell_{\max }$. To compute the total number of evolutions needed we have to take into account the following facts: (i) We need to compute the real and imaginary parts [the source term in the field equations (8) is complex as shown in Eq. [10)] for each $(\ell, m)$ harmonic component of $\Phi^{\ell m}$; (ii) the real character of the scalar field implies that for each $(\ell, m)$ the relation $\bar{\Phi}^{\ell-m}=(-1)^{m} \Phi^{\ell m}$ (we do not need to compute the modes with $m<0$ ) holds; and (iii) For any $(\ell, m)$, we have

$$
Y^{\ell m}(\pi / 2, \varphi)=0 \text { for } \ell+m \text { odd },
$$

and hence, the jumps for these modes are exactly zero [see Eq. (18) and Eqs. (23), (24) and (29), (30)] and we do not need to compute them. Therefore, the total number of evolutions we need to make in terms of $\ell_{\max }$ is

$$
N_{\text {evolutions }}=\frac{\left(\ell_{\max }+1\right)\left(\ell_{\max }+2\right)}{2} .
$$

Once we have evolved the equations for the retarded field we have to regularize it, $\ell$ by $\ell$, by computing the components of the gradient of the singular field at the particle location, either using Eq. (13) or Eq. (B16), and subtracting it from the gradient of the retarded field. In Figure 6 we show the results of the computations of the components of the gradient of the regularized field, which have been obtained by choosing $\ell_{\max }=17$, for which we needed to perform 171 evolutions of the retarded field equations. The particular multidomain framework that 


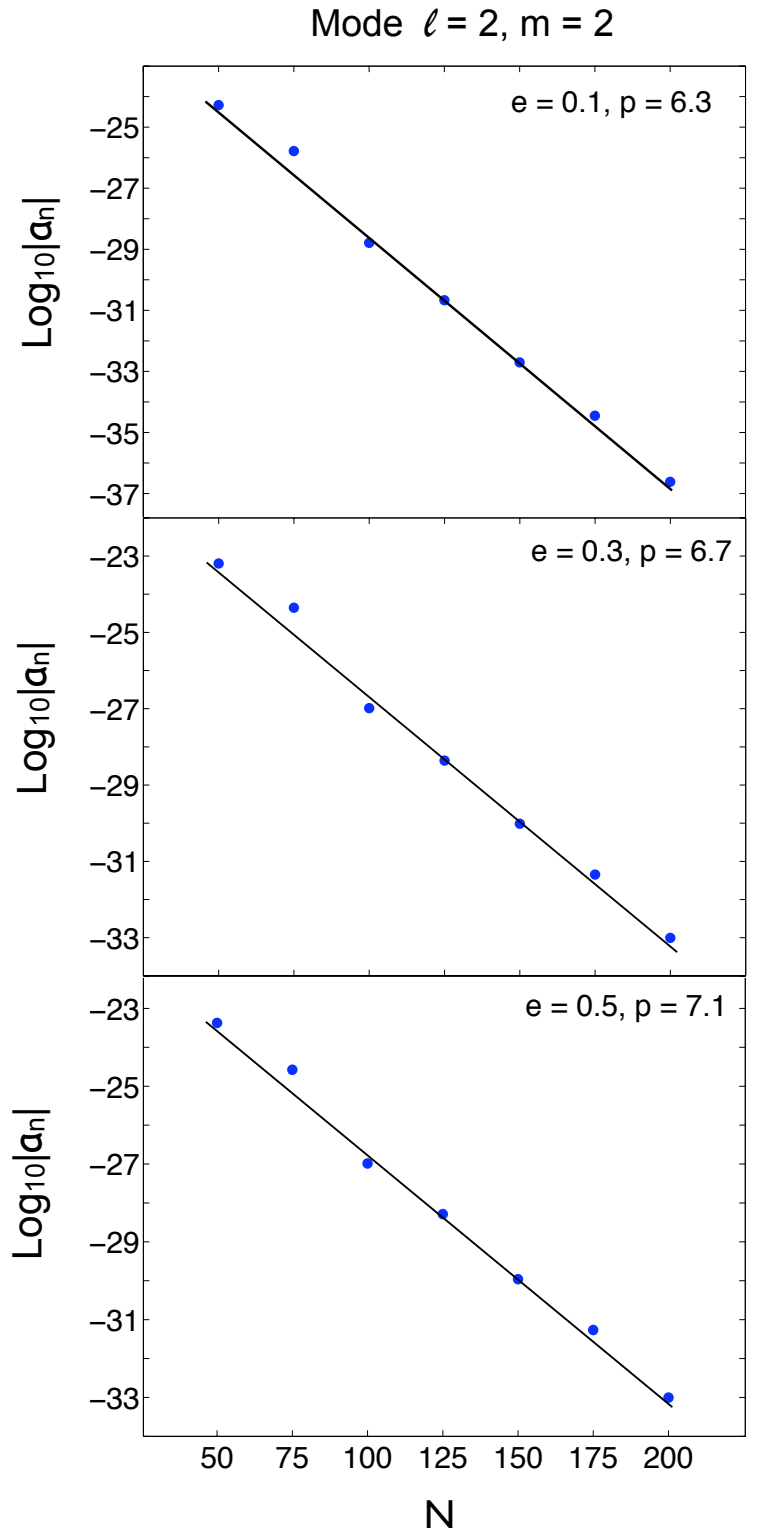

FIG. 4: We show the dependence of the truncation error, as estimated from the quantity $\log _{10}\left|a_{N}\right|$, on the number of collocation points, $N$, for evolutions of the harmonic mode $\ell=$ $m=2$ of the field variable $\psi^{\ell m}$. From top to bottom, we show the truncation error for: (i) $(e, p)=(0.1,6.3)$; (ii) $(e, p)=$ $(0.3,6.7)$; and (iii) $(e, p)=(0.5,7.1)$. The plot corresponds to the solution on the subdomain to the right of the particle. The tortoise radial coordinate size of this subdomain, i.e. $\mid r_{\mathrm{P}+1, \mathrm{~N}}^{*}-$ $r_{p}^{*}(t) \mid$, is in the range $20-40 \mathrm{M}$. The good fit of the data to a straight line confirms the exponential convergence of the numerical computations.

we employed for these computations consists of $D=10$ subdomains and $N=100$ collocation points per subdomain.

Finally, we also want to show some numerical values of the components of the gradient of the regularized field. To that end, we have chosen to evaluate them at the near- est radial location to the pericenter radius [see Eq. A2 ] ] available in our evolutions. In Table [I we show these values for the three orbits of Figure 3 . In order to have some reference to compare these values, we also provide the values for the last stable circular orbit $(r=6 M)$ obtained, using frequency-domain methods, in 61. Our numerical values have been obtained by using a multidomain framework consisting of $D=80$ subdomains and $N=50$ collocation points. Here, we need to make an important comment: In Paper I, where the evolutions were always done using the penalty method, we always quoted two values for each component of the gradient of the regularized field. The reason is that in our framework we have two different nodes for the particle (one that belongs to the subdomain to the left of it, $\Omega_{\mathrm{P}}$, and one that belongs to the subdomain to the right of it, $\Omega_{\mathrm{P}+1}$ ). Since the equations are asymmetric with respect to the particle location, the evolution via penalty method only provides values of the variables at the particle location that are approximately the same but not identical. The advantage of this is that it provides a criterium to decide what is the maximum accuracy of the calculations. In contrast, the new method of evolution proposed in this paper, the direct communication of the characteristic fields, provides, by construction, identical values at both nodes associated with the particle (excepting in the case in which we use spectral filters to eliminate spurious high-frequency modes in the solution, which is not what we have done for the results of this paper). Here, we have presented values obtained with the formulation that uses the direct communication of characteristic fields (which provides values that are the same, up to machine precision, at the two particle nodes), but we have checked that they are consistent with the values obtained by using the penalty method.

\section{CONCLUSIONS AND DISCUSSION}

In this paper we have extended the time-domain techniques introduced in Paper I for the computation of the self-force from the case of circular orbits to generic orbits, what we have called the PwP formulation. The key ingredient of this formulation is to consider a multidomain framework in which the particle is always located at the interface of two subdomains, even in the case of eccentric orbits. In this way, the equations that we have solved at each subdomain are homogeneous wave-type equations for the retarded field, without distributional sources. Then, the solutions of these equations are sufficiently smooth to provide a good convergence of the numerical method involved, in our case the PSC method (which provides exponential convergence for smooth solutions). The way in which we keep the particle at a fixed node is by using a time-dependent mapping between the physical domain (the one described by the Schwarzschild radial coordinate $r$ ) and the spectral domain (the one in which the calculations of the PSC method take place). 

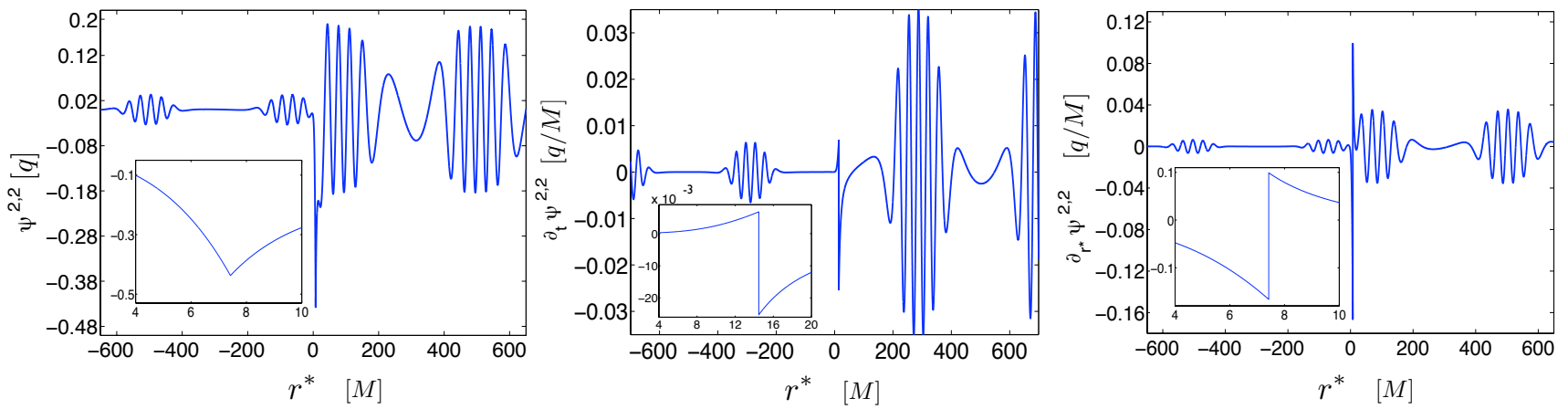

FIG. 5: Snapshots of the evolution of a scalar charged particle in eccentric orbits around a non-rotating MBH. The orbital parameters are $(e, p)=(0.5,7.1)$. The simulations used $D=10$ subdomains and $N=100$ collocation points per subdomain. They show the evolution of the variables $\psi^{\ell m}$ (left), $\phi^{\ell m}=\partial_{t} \psi^{\ell m}$ (center), and $\varphi^{\ell m}=\partial_{r^{*}} \psi^{\ell m}$ (right), for the mode $\ell=m=2$. In particular, they show (see the boxes inside the plots) how the jumps in the time and radial derivatives of the scalar field $\Phi^{\ell m}$ are resolved in the multidomain computational framework that implements the PwP scheme.
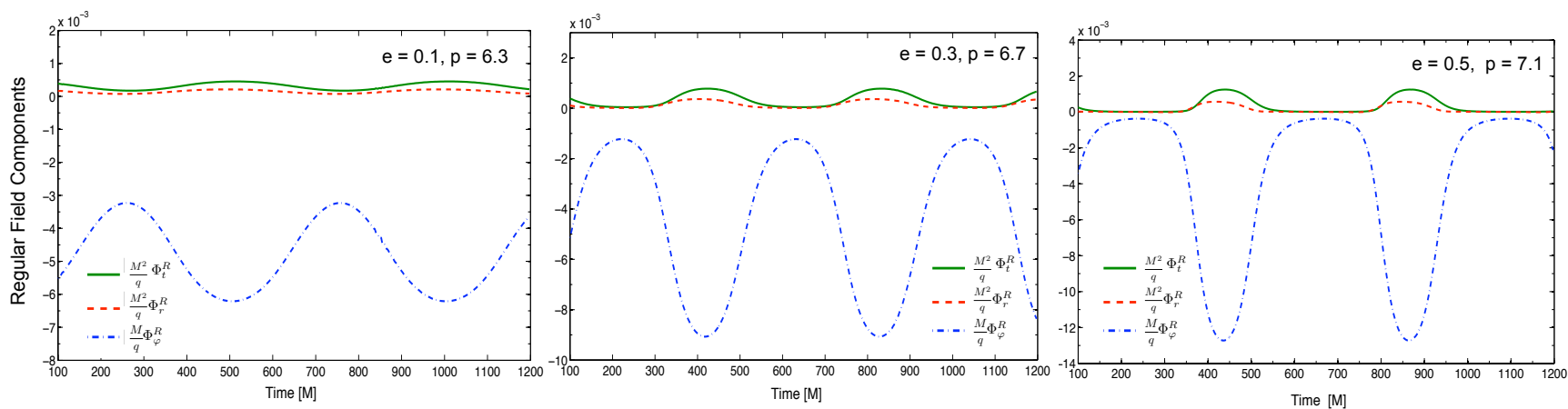

FIG. 6: Evolution of the components of the gradient of the regularized field, $\nabla_{\alpha} \Phi^{\mathrm{R}}$, for a scalar charged particle in eccentric orbits around a non-rotating $\mathrm{MBH}$. From left to right, the orbital parameters of the orbits are: $(\mathrm{i})(e, p)=(0.1,6.3) ;(\mathrm{ii})(e, p)$ $=(0.3,6.7)$; and (iii) $(e, p)=(0.5,7.1)$. For each orbit (frame), the solid line represents the evolution of the dimensionless time component, $\frac{M^{2}}{q} \Phi_{t}^{\mathrm{R}}$; the dashed line represents the evolution of the dimensionless radial component, $\frac{M^{2}}{q} \Phi_{r}^{\mathrm{R}}$; and the dot-dashed line represents the evolution of the dimensionless azimuthal component, $\frac{M}{q} \Phi_{\varphi}^{\mathrm{R}}$. The numerical setup for these calculations used $D=10$ subdomains and $N=100$ collocation points per subdomain.

TABLE I: Numerical values of the components of the gradient of the regularized field at the pericenter radius. We present our estimations for the orbits shown in Figure 3 (i) $(e, p)=(0.1,6.3)$ [Column 3]; (ii) $(e, p)=(0.3,6.7)[$ Column 4]; and (iii) $(e, p)$ $=(0.5,7.1)$ [Column 5]. For reference, we have included the values for circular orbits at the last stable circular orbit $(r=6 M)$ obtained, using frequency-domain methods, in [61] [Column 2].

\begin{tabular}{l|c|c|c|c}
\hline \hline & $(e, p)=(0.0,6.0)$ & $(e, p)=(0.1,6.3)$ & $(e, p)=(0.3,6.7)$ & $(e, p)=(0.5,7.1)$ \\
\hline \hline$r_{\text {peri }}(M)^{a}$ & 6.0 & 5.7272727 & 5.1538461 & 4.7333333 \\
$r_{p}(M)^{b}$ & 6.0 & 5.7272925 & 5.1538801 & 4.7333989 \\
$\frac{M^{2}}{q} \Phi_{t}^{\mathrm{R}}$ & $3.609072 \cdot 10^{-4}$ & $4.5171 \cdot 10^{-4}$ & $7.6980 \cdot 10^{-4}$ & $1.2330 \cdot 10^{-3}$ \\
$\frac{M^{2}}{q} \Phi_{r}^{\mathrm{R}}$ & $1.67728 \cdot 10^{-4}$ & $2.1250 \cdot 10^{-4}$ & $3.6339 \cdot 10^{-4}$ & $5.6122 \cdot 10^{-4}$ \\
$\frac{M}{q} \Phi_{\varphi}^{\mathrm{R}}$ & $-5.304231 \cdot 10^{-3}$ & $-6.2040 \cdot 10^{-3}$ & $-9.0402 \cdot 10^{-3}$ & $-1.2685 \cdot 10^{-2}$ \\
\hline \hline
\end{tabular}

${ }^{a}$ This is the exact value of the pericenter radius for each orbit.

${ }^{b}$ This is the value of the radial coordinate at which the gradient of the regularized field has been evaluated. It is also the nearest value of the radial coordinate to the pericenter value available in our numerical evolutions. 
The details of this mapping are given in Sec. IV] The technical difficulties are then transfered to the communication between the different subdomains, in particular between the subdomains that surround the particle. In this sense, we have extended the use of the penalty method to the eccentric case and proposed a new method for the communication between subdomains based on the transfer of the characteristic fields of the hyperbolic system of equations. These two methods are different enough that their numerical implementation can be considered to lead to different numerical codes, which is good for checking the results of the computations.

The main advantages of the PwP formulation in combination with the PSC numerical method were already described in Paper I and are still valid for what we have presented in this paper, so we do not discuss them further here. Instead, we discuss the possible improvements of the methods presented. In Paper I, we already mentioned a number of such improvements: (i) Compactification of the computational domain, in other to improve the accuracy of the (global) outgoing boundary conditions which will allow longer evolutions maintaining the accuracy. (ii) Reduction of the time step, by abandon the linear mapping between the physical and spectral representations, using a mapping of the type introduced by Kosloff and Tal-Ezer [73. (iii) Richardson extrapolation, to improve the estimations of the values of the self-force by using our analytical knowledge of the expansions in inverse powers of the harmonic number $\ell$, like in [47. These improvements, although they have not been implemented in the present work, can be perfectly applied to our framework and have significant potential to improve the efficiency of the computations. Another improvement that can be even more important than the ones already mentioned has to do with the distribution of subdomains. As it was discussed in Paper I, it is very important to choose appropriately the (tortoise coordinate) size of the subdomains in order to resolve accurately the different harmonic modes. In this sense, the modes with high harmonic number $m$ are more difficult due to the $e^{i m \varphi_{p}(t)}$ dependence of the source term in the original equations [see Eqs. (8) and [10]]. This means that the higher $m$ is, the higher the frequency of the scalar field modes generated is, and hence the shorter the wavelength gets. Therefore, these high $m$ modes require more spatial resolution (also in time). Both in the calculations of Paper I and in this paper we have used a unique subdomain structure for all the modes. Since we must set the size of the subdomains in such a way that the high $m$ modes are properly resolved, this means that we are using excessive resources for the low $m$ modes. Thus, the optimal strategy would be to adapt the subdomain structure to the type of mode we are dealing with. One can improve in this way the efficiency and reduce significantly the computational resources involved.

Another way of improving the performance of the numerical computations is to provide better initial data, or at least to reduce the negative effects of the initial burst produced when we prescribe zero initial data [Eq. [86]]. This is important since as it has been suggested in [74, certain components of this unphysical burst can persist in time, which can deteriorate significantly the precision of the calculations. A possible way of alleviating the situation is to switch on the particle during a certain time scale, by multiplying the source terms (in our case the jumps) by a convenient function of time (starting at zero initially and approaching unity). We have been exploring this possibility, that turns out to be critical in the implementation that makes use of Eqs. (73) and (74), and we will report on this elsewhere.

To finish, the main goal of the formulation presented in this paper is to develop an accurate and efficient method to compute the self-force in situations of physical interest. In particular, for systems of interest for the future observatory LISA. This means to use these techniques in the gravitational case and for spinning MBHs. In this sense, we have to mention that while it is not difficult to transfer the techniques discussed here to the gravitational case, to do the same with the case of a spinning black hole may require new technical improvements which we will the subject of future investigations.

\section{Acknowledgments}

We would like to thank L. Barack, M. Jasiulek, E. Poisson, L. Rezzolla, U. Sperhake, and B. Wardell for helpful discussions and comments. PCM is supported by a predoctoral FPU fellowship of the Spanish Ministry of Science and Innovation (MICINN). CFS acknowledges support from the Ramón y Cajal Programme of the Spanish Ministry of Education and Science (MEC) and by a Marie Curie International Reintegration Grant (MIRG-CT-2007-205005/PHY) within the 7th European Community Framework Programme. JLJ acknowledges a Fellowship from the Alexander von Humboldt Foundation and the hospitaly of the Institute of Space Sciences (CSIC-IEEC) during the realization of this work. Financial support from the contracts ESP2007-61712 (MEC), FIS2008-06078-C03-01 and FIS2008-06078-C0303 (MICINN), and FQM2288 and FQM219 (Junta de Andalucía) is gratefully acknowledged. This research has used the resources of the Centre de Supercomputació de Catalunya (CESCA).

\section{Appendix A: Particle motion}

In this section we summarize the form of the geodesic equations for orbits around a Schwarzschild MBH that we use in this work. Using the constants of motion and the normalization condition of the particle's velocity $u^{\mu}$, $g_{\mu \nu} u^{\mu} u^{\nu}=-1$, we can reduce the geodesic equations to first-order ODEs for $\left(t_{p}, r_{p}, \varphi_{p}\right)$. To avoid the turning points in the integration of the resulting equation for the radial coordinate, we use an angle variable that is related 
to the radial coordinate via

$$
r_{p}=\frac{p M}{1+e \cos \chi_{p}}
$$

where $p$ is the dimensionless semilatus rectum and $e$ is the eccentricity. From this expression, the minimum (pericenter, $r_{\text {peri }}$ ) and maximum (apocenter, $r_{\text {apo }}$ ) values of the radial coordinate, the turning points, are

$$
r_{\text {peri }}=\frac{p M}{1+e}, \quad r_{\text {apo }}=\frac{p M}{1-e} .
$$

Since the spacetime is static and spherically symmetric, the geodesics have the following constants of motion: En- ergy $E_{p}=-u_{t}$ and angular momentum $L_{p}=u_{\phi}$, which can be written in terms of the orbital parameters $(p, e)$ as

$$
\begin{aligned}
E_{p}^{2} & =\frac{(p-2-2 e)(p-2+2 e)}{p\left(p-3-e^{2}\right)} \\
L_{p}^{2} & =\frac{p^{2} M^{2}}{p-3-e^{2}} .
\end{aligned}
$$

The equations for $\left(\chi_{p}(t), \varphi_{p}(t)\right)$ are

$$
\begin{aligned}
\frac{d \chi_{p}}{d t} & =\frac{\left(p-2-2 e \cos \chi_{p}\right) \sqrt{p-6-2 e \cos \chi_{p}}\left(1+e \cos \chi_{p}\right)^{2}}{M p^{2} \sqrt{(p-2)^{2}-4 e^{2}}} \\
\frac{d \varphi_{p}}{d t} & =\frac{\left(p-2-2 e \cos \chi_{p}\right)\left(1+e \cos \chi_{p}\right)^{2}}{M p^{3 / 2} \sqrt{(p-2)^{2}-4 e^{2}}}
\end{aligned}
$$

Two additional expressions of interest for this paper are the first and second time derivatives of the tortoise coordinate, $\dot{r}_{p}^{*}$ and $\ddot{r}_{p}^{*}$. They can be obtained from the geodesic equations doing appropriate manipulations. The result is

$$
\dot{r}_{p}^{*}=\sqrt{1-\frac{f_{p}}{E_{p}^{2}}\left(1+\frac{L_{p}^{2}}{r_{p}^{2}}\right)},
$$

and

$$
\ddot{r}_{p}^{*}=-\frac{f_{p}}{r_{p} E_{p}^{2}}\left\{\frac{M}{r_{p}}-\frac{L_{p}^{2}}{r_{p}^{2}}\left(1-\frac{3 M}{r_{p}}\right)\right\}
$$

\section{Appendix B: Structure of the Singular Field}

In the description of the problem in Sec. II it was made clear that the computation of the self-force requires the regularization of the retarded scalar field. This can be done in the framework of the mode-sum scheme, where we need to compute both the harmonic components of the retarded and singular parts of the scalar field. The singular field near the particle has a particular dependence on the harmonic number $\ell$, which is fully determined by the specification of a set of regularization parameters (which can be derived by analyzing the field equations in a worldtube close to the particle's worldline). In this Appendix we summarize the expressions for these parameters that we have used in this work (see [46, 48, 63, 64]).

We start with the prescription introduced by Haas and Poisson [48], which is the only one that includes the regularization parameter that goes with the $\ell^{-2}$ (for large $\ell$ ) behaviour of the singular scalar field, namely $D_{\alpha}$. This term, although it is not necessary for the computation of the regularized field, it is useful in order to accelerate the convergence of it as we increase the value of $\ell_{\max }$ (the maximum $\ell$ harmonic number that contributes to the computation of the regularized field, and hence to the self-force). Following [48] we decompose $\nabla_{\nu} \Phi$ in terms of a complex tetrad $e_{(\mu)}^{\alpha}(x)$, with the index $(\mu)=\{(0),(+),(-),(3)\}$ labeling the different components of this tetrad

$$
\begin{aligned}
e_{(0)}^{\alpha} & =\left[\frac{1}{\sqrt{f}}, 0,0,0\right] \\
e_{(3)}^{\alpha} & =\left[0, \sqrt{f} \cos \theta,-\frac{\sin \theta}{r}, 0\right] \\
e_{( \pm)}^{\alpha} & =\left[0, \sqrt{f} \sin \theta e^{ \pm i \varphi}, \frac{\cos \theta e^{ \pm i \varphi}}{r}, \frac{ \pm i e^{ \pm i \varphi}}{r \sin \theta}\right]
\end{aligned}
$$

The components of the gradient of the retarded field in this tetrad, $\Phi_{(\mu)} \equiv \Phi_{\alpha} e_{(\mu)}^{\alpha}$, are scalars. Thus, we can expand $\Phi_{(\mu)}$ in scalar spherical harmonics:

$$
\Phi_{(\mu)}\left(x^{\alpha}\right)=\sum_{\ell=0}^{\infty} \sum_{m=-\ell}^{\ell} \Phi_{(\mu)}^{\ell m}(t, r) Y^{\ell m}(\theta, \varphi) .
$$

Then, the components of the gradient of the regular field [Eq. [11] ] are given by

$$
\Phi_{\alpha}^{\mathrm{R}}=\Phi_{\alpha}-\Phi_{\alpha}^{\mathrm{S}}=\left\{\Phi_{(\mu)}-\Phi_{(\mu)}^{\mathrm{S}}\right\} e_{\alpha}^{(\mu)},
$$

where, $\Phi_{(\mu)}$ and $\Phi_{(\mu)}^{S}$, are the tetrad projections of the gradient of the retarded and singular fields. Then, the 
relation between the tetrad and coordinate components of the gradient of the regular field can be written in the form:

$$
\begin{aligned}
\Phi_{(0)}^{\ell m} & =\frac{1}{\sqrt{f}} \partial_{t} \Phi^{\ell m}, \\
\Phi_{(+)}^{\ell m} & =-\hat{\alpha}_{(+)}^{\ell m}\left(\Phi^{\ell-1 m-1}\right)+\hat{\beta}_{(+)}^{\ell m}\left(\Phi^{\ell+1 m-1}\right), \\
\Phi_{(-)}^{\ell m} & =\hat{\alpha}_{(-)}^{\ell m}\left(\Phi^{\ell-1 m+1}\right)-\hat{\beta}_{(-)}^{\ell m}\left(\Phi^{\ell+1 m+1}\right), \\
\Phi_{(3)}^{\ell m} & =\hat{\alpha}_{3}^{\ell m}\left(\Phi^{\ell-1 m}\right)+\hat{\beta}_{3}^{\ell m}\left(\Phi^{\ell+1 m}\right),
\end{aligned}
$$

where $\hat{\alpha}_{(+)}^{\ell m}, \hat{\beta}_{(+)}^{\ell m}, \hat{\alpha}_{(-)}^{\ell m}, \hat{\beta}_{(-)}^{\ell m}, \hat{\alpha}_{3}^{\ell m}$, and $\hat{\beta}_{3}^{\ell m}$ are linear operators given by the following expressions

$$
\begin{gathered}
\hat{\alpha}_{(+)}^{\ell m}=\sqrt{\frac{(\ell+m-1)(\ell+m)}{(2 \ell-1)(2 \ell+1)}}\left(\sqrt{f} \frac{\partial}{\partial r}-\frac{\ell-1}{r}\right)(\mathrm{B} 10)(\mathrm{B} 11) \\
\hat{\alpha}_{(-)}^{\ell m}=\sqrt{\frac{(\ell-m-1)(\ell-m)}{(2 \ell-1)(2 \ell+1)}}\left(\sqrt{f} \frac{\partial}{\partial r}-\frac{\ell-1}{r}\right) \\
\left.\hat{\alpha}_{3}=\sqrt{\frac{(\ell-m)(\ell+m)}{(2 \ell-1)(2 \ell+1)}(\sqrt{f}} \frac{\partial}{\partial r}-\frac{\ell-1}{r}\right), \quad(\mathrm{B} 12) \\
\hat{\beta}_{(+)}^{\ell m}=\sqrt{\frac{(\ell-m+1)(\ell-m+2)}{(2 \ell+1)(2 \ell+3)}}\left(\sqrt{f} \frac{\partial}{\partial r}+\frac{\ell+2}{r}\right)_{\mathrm{B}, 13)} \\
\hat{\beta}_{3}^{\ell m}=\sqrt{\frac{(\ell-m+1)(\ell+m+1)}{(2 \ell+1)(2 \ell+3)}}\left(\sqrt{f} \frac{\partial}{\partial r}+\frac{\ell+2}{r}\right)(\mathrm{B} 1 \\
\hat{\beta}_{(-)}=
\end{gathered}
$$

On the other hand, the tetrad projections of the singular field can be written [in a similar way as in the coordinate case of Eq. [13] ] as

$$
\begin{aligned}
\Phi_{(\mu)}^{\mathrm{S}, \ell} & =q\left\{\left(\ell+\frac{1}{2}\right) A_{(\mu)}+B_{(\mu)}+\frac{C_{(\mu)}}{\ell+\frac{1}{2}}\right. \\
& \left.+\frac{D_{(\mu)}}{\left(\ell-\frac{1}{2}\right)\left(\ell+\frac{3}{2}\right)}+\cdots\right\} .
\end{aligned}
$$

The components $A_{(\mu)}$ of the regularization parameters are given by

$$
\begin{aligned}
& A_{(0)}=\frac{\epsilon_{p} E_{p} \dot{r}_{p}}{f_{p}^{3 / 2}\left(r_{p}^{2}+L_{p}^{2}\right)}, \\
& A_{(+)}=\bar{A}_{(-)}=-\epsilon_{p} \frac{E_{p} e^{i \varphi_{p}}}{\sqrt{f_{p}}\left(r_{p}^{2}+L_{p}^{2}\right)}, \\
& A_{(3)}=0,
\end{aligned}
$$

where we recall that a dot means differentiation with respect to the coordinate time $t$ and $\epsilon_{p}$ is

$$
\epsilon_{p}=\operatorname{sign}\left(r-r_{p}\right)= \begin{cases}1 & \text { if } r>r_{p} \\ -1 & \text { if } r<r_{p}\end{cases}
$$

The components $B_{(\mu)}$ of the regularization parameters are

$$
B_{(0)}=\frac{E_{p}^{2} r_{p} \dot{r}_{p}}{2\left[f_{p}\left(r_{p}^{2}+L_{p}^{2}\right)\right]^{3 / 2}}\left(F_{1 / 2}-2 F_{-1 / 2}\right),
$$

where $F_{1 / 2}$ and $F_{-1 / 2}$ are objects defined in terms of hypergeometric functions 75 as

$$
\begin{gathered}
F_{1 / 2}=F\left(\frac{1}{2}, \frac{1}{2} ; 1 ; \frac{L_{p}^{2}}{r_{p}^{2}+L_{p}^{2}}\right), \\
F_{-1 / 2}=F\left(-\frac{1}{2}, \frac{1}{2} ; 1 ; \frac{L_{p}^{2}}{r_{p}^{2}+L_{p}^{2}}\right),
\end{gathered}
$$

and

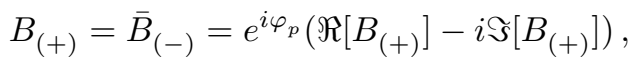

where $\Re(z)$ and $\Im(z)$ denote the real and imaginary parts of the complex number $z$. In the case of $B_{(+)}$, these parts are given by

$$
\begin{gathered}
\Re\left[B_{(+)}\right]=\frac{E_{p}^{2} r_{p} \dot{r}_{p}^{2}}{2 f_{p}^{5 / 2}\left(r_{p}^{2}+L_{p}^{2}\right)^{3 / 2}}\left(2 F_{-1 / 2}-F_{1 / 2}\right)+\frac{1}{2 r_{p} \sqrt{r_{p}^{2}+L_{p}^{2}}}\left(\sqrt{f_{p}} F_{-1 / 2}-2\left(\sqrt{f_{p}}-1\right) F_{1 / 2}\right) \\
\Im\left[B_{(+)}\right]=\frac{\left(2-\sqrt{f_{p}}\right) E_{p} \dot{r}_{p}}{2 L_{p} f_{p}^{3 / 2} \sqrt{\left(r_{p}^{2}+L_{p}^{2}\right)}}\left(F_{1 / 2}-F_{-1 / 2}\right) .
\end{gathered}
$$


Moreover, we have

$$
B_{(3)}=0
$$

and

$$
C_{(\mu)}=0
$$

Finally, the components $D_{(\mu)}$ of the regularization parameters are

$$
\begin{aligned}
D_{(0)} & =\frac{E_{p}^{4} r_{p}^{3} \dot{r}_{p}^{3}}{16\left[f_{p}\left(r_{p}^{2}+L_{p}^{2}\right)\right]^{7 / 2}}\left\{\left(5 r_{p}^{2}-3 L_{p}^{2}\right) F_{1 / 2}-8\left(r_{p}^{2}-L_{p}^{2}\right) F_{-1 / 2}\right\}+\frac{E_{p}^{2} \dot{r}_{p}}{16 f_{p}^{3 / 2}\left(r_{p}^{2}+L_{p}^{2}\right)^{5 / 2}\left\{\left(F_{1 / 2}-F_{-1 / 2}\right) r_{p}^{3}\right.} \\
& +M r_{p}^{2}\left(16 F_{1 / 2}-30 F_{-1 / 2}\right)-r_{p} L_{p}^{2}\left(3 F_{1 / 2}-7 F_{-1 / 2}\right)+M L_{p}^{2}\left(42 F_{1 / 2}-114 F_{-1 / 2}\right) \\
& \left.+\frac{M L_{p}^{4}}{r_{p}^{2}}\left(18 F_{1 / 2}-104 F_{-1 / 2}\right)-\frac{36 M L_{p}^{6}}{r_{p}^{4}} F_{-1 / 2}\right\}
\end{aligned}
$$$$
D_{(+)}=\bar{D}_{(-)}=e^{i \varphi_{p}}\left(\Re\left[D_{(+)}\right]-i \Im\left[D_{(+)}\right]\right), \quad \text { (B30) with }
$$

$$
\begin{aligned}
& \Re\left[D_{(+)}\right]=\frac{E_{p}^{4} r_{p}^{3} \dot{r}_{p}^{4}}{16 f_{p}^{9 / 2}\left(r_{p}^{2}+L_{p}^{2}\right)^{7 / 2}}\left\{8\left(r_{p}^{2}-L_{p}^{2}\right) F_{-1 / 2}-\left(5 r_{p}^{2}-3 L_{p}^{2}\right) F_{1 / 2}\right\}+\frac{E_{p}^{2} r_{p} \dot{r}_{p}^{2}}{8 f_{p}^{2}\left(r_{p}^{2}+L_{p}^{2}\right)^{3 / 2}}\left(F_{1 / 2}-2 F_{-1 / 2}\right) \\
& +\frac{E_{p}^{2} \dot{r}_{p}^{2}}{16 f_{p}^{5 / 2}\left(r_{p}^{2}+L_{p}^{2}\right)^{5 / 2}}\left\{4\left(3 r_{p}^{3}+6 M r_{p}^{2}-L_{p}^{2} r_{p}+31 M L_{p}^{2}+\frac{26 M L_{p}^{4}}{r_{p}^{2}}+\frac{9 M L_{p}^{6}}{r_{p}^{4}}\right) F_{-1 / 2}\right. \\
& \left.-\left(7 r_{p}^{3}+12 M r_{p}^{2}-L_{p}^{2} r_{p}+46 M L_{p}^{2}+\frac{18 M L_{p}^{4}}{r_{p}^{2}}\right) F_{1 / 2}\right\} \\
& +\frac{\sqrt{f_{p}}}{16\left(r_{p}^{2}+L_{p}^{2}\right)^{3 / 2}}\left\{\left(3 r_{p}+8 M+\frac{L_{p}^{2}}{r_{p}}+\frac{26 M L_{p}^{2}}{r_{p}^{2}}+\frac{22 M L_{p}^{4}}{r_{p}^{4}}+\frac{8 M L_{p}^{6}}{r_{p}^{6}}\right) F_{-1 / 2}\right. \\
& \left.-\left(7 r_{p}+6 M+\frac{6 L_{p}^{2}}{r_{p}}+\frac{12 M L_{p}^{2}}{r_{p}^{2}}+\frac{4 M L_{p}^{4}}{r_{p}^{4}}\right) F_{1 / 2}\right\} \\
& -\frac{1}{8 \sqrt{r_{p}^{2}+L_{p}^{2}}}\left\{\left(\frac{1}{r_{p}}+\frac{2 M}{r_{p}^{2}}-\frac{4 M L_{p}^{2}}{r_{p}^{4}}\right) F_{-1 / 2}-\frac{3 F_{1 / 2}}{r_{p}}\right\} \\
& \Im\left[D_{(+)}\right]=\frac{\left(\sqrt{f_{p}}-2\right) E_{p}^{3} r_{p}^{2} \dot{r}_{p}^{3}}{16 L_{p} f_{p}^{7 / 2}\left(r_{p}^{2}+L_{p}^{2}\right)^{5 / 2}}\left\{\left(r_{p}^{2}-7 L_{p}^{2}\right) F_{-1 / 2}-\left(r_{p}^{2}-3 L_{p}^{2}\right) F_{1 / 2}\right\} \\
& +\frac{E_{p} \dot{r}_{p}}{16 L_{p} f_{p}\left(r_{p}^{2}+L_{p}^{2}\right)^{3 / 2}}\left\{\left(4 r_{p}^{2}+2 M r_{p}+7 L_{p}^{2}+\frac{10 M L_{p}^{2}}{r_{p}}+\frac{14 M L_{p}^{4}}{r_{p}^{3}}\right) F_{1 / 2}\right. \\
& \left.-2\left(2 r_{p}^{2}+M r_{p}+5 L_{p}^{2}+\frac{10 M L_{p}^{2}}{r_{p}}+\frac{29 M L_{p}^{4}}{r_{p}^{3}}+\frac{14 M L_{p}^{6}}{r_{p}^{5}}\right) F_{-1 / 2}\right\} \\
& +\frac{E_{p} \dot{r}_{p}}{8 L_{p} f_{p}^{3 / 2}\left(r_{p}^{2}+L_{p}^{2}\right)^{3 / 2}}\left\{2\left(r_{p}^{2}-M r_{p}+4 L_{p}^{2}-\frac{5 M L_{p}^{2}}{r_{p}}+\frac{2 M L_{p}^{4}}{r_{p}^{3}}\right) F_{-1 / 2}\right. \\
& \left.-\left(2 r_{p}^{2}-2 M r_{p}+5 L_{p}^{2}-\frac{8 M L_{p}^{2}}{r_{p}}\right) F_{1 / 2}\right\}
\end{aligned}
$$


and

$$
D_{(3)}=0 \text {. }
$$

On the other hand, the regularization parameters associated with the coordinate expression of the singular field [see Eq. [13]], given in [46, 63, 64, are given by

$$
\begin{gathered}
A_{t}=-\epsilon_{p} \frac{E_{p} \dot{r}_{p}}{f_{p}\left(r_{p}^{2}+L_{p}^{2}\right)}, \\
A_{r}=\epsilon_{p} \frac{E_{p}}{f_{p}\left(r_{p}^{2}+L_{p}^{2}\right)}, \\
A_{\varphi}=0 \\
B_{t}=\frac{E_{p}^{2} r_{p} \dot{r}_{p}\left(F_{1 / 2}-2 F_{-1 / 2}\right)}{2 f_{p}\left(r_{p}^{2}+L_{p}^{2}\right)^{3 / 2}} \\
B_{r}=\frac{E_{p}^{2} r_{p}\left[\left(\dot{r}_{p}^{2}-2 f_{p}^{2}\right) F_{1 / 2}+\left(\dot{r}_{p}^{2}-f_{p}^{2}\right) F_{-1 / 2}\right]}{2 f_{p}^{3}\left(r_{p}^{2}+L_{p}^{2}\right)^{3 / 2}},
\end{gathered}
$$

$$
B_{\varphi}=\frac{E_{p} r_{p} \dot{r}_{p}\left(F_{1 / 2}-F_{-1 / 2}\right)}{2 L_{p} f_{p} \sqrt{r_{p}^{2}+L_{p}^{2}}}
$$

$$
C_{\alpha}=0
$$

In this case, the regularization parameters $D_{\alpha}$ have not been computed.

\section{Appendix C: Time Derivatives of the Jumps in the Characteristic Variables}

The time derivatives of the jumps of the characteristic fields, are given by the following expressions:

$$
\begin{aligned}
\frac{d[U]_{p}}{d T} & =-\frac{4 \pi q}{E_{p} r_{p}^{2}} \frac{f_{p}}{1-\dot{r}_{p}^{*}}\left\{\left[\left(1-\frac{4 M}{r_{p}}\right) \dot{r}_{p}^{*}-\frac{r_{p} \ddot{r}_{p}^{*}}{1-\dot{r}_{p}^{*}}\right] \Re\left[Y^{\ell, m}\right]+m \frac{L_{p} f_{p}}{E_{p} r_{p}} \Im\left[Y^{\ell, m}\right]\right\} \\
& +i\left[\frac{4 \pi q}{E_{p} r_{p}^{2}} \frac{f_{p}}{1-\dot{r}_{p}^{*}}\left\{\left[\left(1-\frac{4 M}{r_{p}}\right) \dot{r}_{p}^{*}-\frac{r_{p} \ddot{r}_{p}^{*}}{1-\dot{r}_{p}^{*}}\right] \Im\left[Y^{\ell, m}\right]-m \frac{L_{p} f_{p}}{E_{p} r_{p}} \Re\left[Y^{\ell, m}\right]\right\}\right], \\
\frac{d[V]_{p}}{d T} & =\frac{4 \pi q}{E_{p} r_{p}^{2}} \frac{f_{p}}{1+\dot{r}_{p}^{*}}\left\{\left[\left(1-\frac{4 M}{r_{p}}\right) \dot{r}_{p}^{*}+\frac{r_{p} \ddot{r}_{p}^{*}}{1+\dot{r}_{p}^{*}}\right] \Re\left[Y^{\ell, m}\right]+m \frac{L_{p} f_{p}}{E_{p} r_{p}} \Im\left[Y^{\ell, m}\right]\right\} \\
& -i\left[\frac{4 \pi q}{E_{p} r_{p}^{2}} \frac{f_{p}}{1+\dot{r}_{p}^{*}}\left\{\left[\left(1-\frac{4 M}{r_{p}}\right) \dot{r}_{p}^{*}-\frac{r_{p} \ddot{r}_{p}^{*}}{1+\dot{r}_{p}^{*}}\right] \Im\left[Y^{\ell, m}\right]-m \frac{L_{p} f_{p}}{E_{p} r_{p}} \Re\left[Y^{\ell, m}\right]\right\}\right],
\end{aligned}
$$

where $\Re\left[Y^{\ell, m}\right]$ and $\Im\left[Y^{\ell, m}\right]$ are the real and imaginary parts of the scalar spherical harmonic $Y^{\ell m}$, and $\dot{r}_{p}^{*}$ and $\ddot{r}_{p}^{*}$ are given by Eqs. A7 and $\mathrm{A} 8$ respectively.
[1] Laser Interferometer Gravitational-Wave Observatory (LIGO), URL: www.ligo.caltech.edu.

[2] GEO600, URL: www.geo600.org.

[3] VIRGO, URL: www .virgo.infn.it.

[4] Large Scale Cryogenic Gravitational Wave Telescope (LCGT), URL: gw.icrr.u-tokyo.ac.jp/lcgt.

[5] Laser Interferometer Space Antenna (LISA), URLs: www.esa.int, lisa.jpl.nasa.gov.

[6] Einstein Telescope, URL: www.et-gw.eu.
[7] A. Lobo and C. F. Sopuerta, eds., Laser Interferometer Space Antenna. Proceedings of the 7th International LISA Symposium, vol. 26 (Classical and Quantum Gravity, Institute of Physics, 2009).

[8] A. Lobo and C. F. Sopuerta, eds., Laser Interferometer Space Antenna. Proceedings of the 7th International LISA Symposium, vol. 154 (Journal of Physics: Conference Series, Institute of Physics, 2009).

[9] P. Amaro-Seoane et al., Class. Quant. Grav. 24, R113 
(2007), astro-ph/0703495

[10] L. S. Finn and K. S. Thorne, Phys. Rev. D62, 124021 (2000), gr-qc/0007074.

[11] Y. Mino, M. Sasaki, and T. Tanaka, Phys. Rev. D55, 3457 (1997), gr-qc/9606018.

[12] T. C. Quinn and R. M. Wald, Phys. Rev. D56, 3381 (1997), gr-qc/9610053.

[13] S. E. Gralla and R. M. Wald, Class. Quant. Grav. 25, 205009 (2008), 0806.3293.

[14] A. Pound, Phys. Rev. D81, 024023 (2010), 0907.5197.

[15] A. Pound (2010), 1003.3954.

[16] K. Glampedakis, Class. Quant. Grav. 22, S605 (2005), gr-qc/0509024.

[17] E. Poisson, Living Rev. Relativity 7, 6 (2004), gr-qc/0306052, URL http://www.livingreviews.org/ lrr-2004-6.

[18] L. Barack (2009), 0908.1664v2, URL http://arXiv.org/ abs/0908.1664v2

[19] L. Barack and C. Cutler, Phys. Rev. 69, 082005 (2004), gr-qc/0310125.

[20] S. Babak, H. Fang, J. R. Gair, K. Glampedakis, and S. A. Hughes, Phys. Rev. D75, 024005 (2007), gr-qc/0607007.

[21] S. Drasco and S. A. Hughes, Phys. Rev. D73, 024027 (2006), gr-qc/0509101.

[22] N. Yunes, A. Buonanno, S. A. Hughes, M. Coleman Miller, and Y. Pan, Phys. Rev. Lett. 104, 091102 (2010), 0909.4263.

[23] J. R. Gair, L. Barack, T. Creighton, C. Cutler, S. L. Larson, E. S. Phinney, and M. Vallisneri, Class. Quant. Grav. 21, S1595 (2004), gr-qc/0405137.

[24] C. Hopman and T. Alexander, Astrophys. J. 645, L133 (2006), astro-ph/0603324.

[25] B. F. Schutz, Class. Quant. Grav. 26, 094020 (2009).

[26] C. L. MacLeod and C. J. Hogan, Phys. Rev. D77, 043512 (2008), 0712.0618.

[27] J. R. Gair, Class. Quant. Grav. 26, 094034 (2009), 0811.0188

[28] N. A. Collins and S. A. Hughes, Phys. Rev. D69, 124022 (2004), gr-qc/0402063.

[29] K. Glampedakis and S. Babak, Class. Quant. Grav. 23, 4167 (2006), gr-qc/0510057.

[30] L. Barack and C. Cutler, Phys. Rev. D75, 042003 (2007), gr-qc/0612029.

[31] N. Yunes, C. F. Sopuerta, L. J. Rubbo, and K. HolleyBockelmann, Astrophys. J. 675, 604 (2008), 0704.2612.

[32] J. Brink, Phys. Rev. D78, 102001 (2008), 0807.1178.

[33] S. J. Vigeland and S. A. Hughes, Phys. Rev. D81, 024030 (2010), 0911.1756.

[34] S. A. Hughes (2010), 1002.2591.

[35] S. A. Hughes, AIP Conf. Proc. 873, 233 (2006), grqc/0608140.

[36] C. F. Sopuerta and N. Yunes, Phys. Rev. D80, 064006 (2009), 0904.4501.

[37] M. C. Miller and E. J. M. Colbert, Int. J. Mod. Phys. D13, 1 (2004), astro-ph/0308402.

[38] M. C. Miller (2008), 0812.3028.

[39] D. A. Brown et al., Phys. Rev. Lett. 99, 201102 (2007), gr-qc/0612060.

[40] I. Mandel, D. A. Brown, J. R. Gair, and M. C. Miller (2007), 0705.0285.

[41] L. Barack and A. Ori, Phys. Rev. D61, 061502 (2000), gr-qc/9912010.

[42] L. Barack, Phys. Rev. D62, 084027 (2000), grqc/0005042.
[43] L. Barack, Phys. Rev. D64, 084021 (2001), grqc/0105040.

[44] Y. Mino, H. Nakano, and M. Sasaki, Prog. Theor. Phys. 108, 1039 (2002), gr-qc/0111074.

[45] L. Barack, Y. Mino, H. Nakano, A. Ori, and M. Sasaki, Phys. Rev. Lett. 88, 091101 (2002), gr-qc/0111001.

[46] L. Barack and A. Ori, Phys. Rev. D66, 084022 (2002), gr-qc/0204093.

[47] S. Detweiler, E. Messaritaki, and B. F. Whiting, Phys. Rev. D67, 104016 (2003), gr-qc/0205079.

[48] R. Haas and E. Poisson, Phys. Rev. D74, 044009 (2006), gr-qc/0605077.

[49] C. F. Sopuerta, P. Sun, P. Laguna, and J. Xu, Class. Quantum Grav. 23, 251 (2006), gr-qc/0507112.

[50] L. Barack and D. A. Golbourn, Phys. Rev. D76, 044020 (2007), 0705.3620.

[51] L. Barack, D. A. Golbourn, and N. Sago, Phys. Rev. D76, 124036 (2007), 0709.4588.

[52] I. Vega and S. Detweiler, Phys. Rev. D77, 084008 (2008), 0712.4405 .

[53] C. O. Lousto and H. Nakano, Class. Quant. Grav. 25, 145018 (2008), 0802.4277.

[54] S. Brandt and B. Bruegmann, Phys. Rev. Lett. 78, 3606 (1997), gr-qc/9703066.

[55] M. Campanelli, C. O. Lousto, P. Marronetti, and Y. Zlochower, Phys. Rev. Lett. 96, 111101 (2006), grqc/0511048.

[56] J. G. Baker, J. Centrella, D.-I. Choi, M. Koppitz, and J. van Meter, Phys. Rev. Lett. 96, 111102 (2006), grqc/0511103.

[57] P. Canizares and C. F. Sopuerta, J. Phys. Conf. Ser. 154, 012053 (2009), 0811.0294.

[58] P. Canizares and C. F. Sopuerta, Phys. Rev. D79, 084020 (2009), 0903.0505.

[59] S. E. Field, J. S. Hesthaven, and S. R. Lau, Class. Quant. Grav. 26, 165010 (2009), 0902.1287.

[60] R. Haas, Phys. Rev. D75, 124011 (2007), 0704.0797.

[61] L. M. Diaz-Rivera, E. Messaritaki, B. F. Whiting, and S. Detweiler, Phys. Rev. D70, 124018 (2004), grqc/0410011.

[62] S. Detweiler and B. F. Whiting, Phys. Rev. D67, 024025 (2003), gr-qc/0202086.

[63] D.-H. Kim (2004), gr-qc/0402014.

[64] D.-H. Kim, Ph.D. thesis, University of Florida (2005).

[65] R. Courant and D. Hilbert, Methods of Mathematical Physics, vol. II. Partial Differential Equations (Interscience Publishers, John Wiley \& Sons, New York, 1966).

[66] C. F. Sopuerta and P. Laguna, Phys. Rev. D73, 044028 (2006), gr-qc/0512028.

[67] H. P. Pfeiffer, L. E. Kidder, M. A. Scheel, and S. A. Teukolsky, Comput. Phys. Commun. 152, 253 (2003), gr-qc/0202096.

[68] J. P. Boyd, Chebyshev and Fourier Spectral Methods (Dover, New York, 2001), 2nd ed.

[69] J. S. Hesthaven, Applied Numerical Mathematics 33, 23 (2000).

[70] B. Gustafsson, H. Kreiss, and J. Oliger, Time dependent problems (John Wiley \& Sons, New York, 1995).

[71] J. C. Butcher, Numerical Methods for Ordinary Differential Equations (John Wiley \& Sons, Chichester, 2008), 2nd ed.

[72] W. H. Press, B. P. Flannery, S. A. Teukolsky, and W. T. Vetterling, Numerical Recipes: The Art of Scientific Computing (Cambridge University Press, Cam- 
bridge, 1992).

[73] D. Kosloff and H. Tal-Ezer, Journal of Computational Physics 104, 457 (1993).

[74] S. E. Field, J. S. Hesthaven, and S. R. Lau (2010), 1001.2578.

[75] M. Abramowitz and I. A. Stegun, Handbook of Mathe- matical Functions with Formulas, Graphs, and Mathematical Tables (Dover, New York, 1972).

[76] Notice that in Paper I there is a typo in the formula for the source term, it contains $f^{2}\left(r_{p}\right)$ instead of $f\left(r_{p}\right)$. 